\title{
Objective and subjective assessment of sound attenuation efficiency by individual hearing protectors with various acoustic filters - a preliminary study
}

\section{Roman Gołębiewski}

Uniwersytet im Adama Mickiewicza w Poznaniu Wydzial Fizyki

Andrzej Wicher ( $\square$ awaku@amu.edu.pl )

Adam Mickiewicz University https://orcid.org/0000-0003-4332-6564

Artur Duraj

Uniwersytet im Adama Mickiewicza w Poznaniu Wydzial Fizyki

Milena Kaczmarek-Klinowska

Uniwersytet im Adama Mickiewicza w Poznaniu Wydzial Fizyki

Karina Mrugalska-Handke

Uniwersytet im Adama Mickiewicza w Poznaniu Wydzial Fizyki

\section{Research}

Keywords: hearing protector, earplug, acoustic filter, hearing loss, artificial head

Posted Date: March 9th, 2020

DOI: https://doi.org/10.21203/rs.3.rs-16338/v1

License: (c) (1) This work is licensed under a Creative Commons Attribution 4.0 International License.

Read Full License 
Objective and subjective assessment of sound attenuation efficiency by individual hearing protectors with various acoustic filters - a preliminary study

Roman Gołębiewski, Andrzej Wicher *, Artur Duraj, Milena Kaczmarek - Klinowska, Karina Mrugalska - Handke

Department of Acoustics, Faculty of Physics, Adam Mickiewicz University Uniwersytetu Poznańskiego 2, 61-614 Poznań, Poland

${ }^{*}$ Corresponding author:

Andrzej Wicher, e-mail: awaku@amu.edu.pl,

Department of Acoustics, Faculty of Physics, Adam Mickiewicz University Uniwersytetu Poznańskiego 2, 61-614 Poznań, Poland 


\begin{abstract}
Background: Hearing loss caused by excessive noise levels is one of the most common health risks for employees. One solution for noise reduction is the use of hearing protectors, which is one of the most effective methods for protecting hearing from noise at the workplace. In order to obtain different attenuation efficiency, individual hearing protectors can be equipped with a suitable acoustic filter. The effectiveness of the hearing protectors attenuation is based on real measurement of hearing thresholds for normal-hearing people with and without hearing protectors. However, this is a time-consuming process and the obtained values are characterized by quite large inter-individual variability. The optimal solution is to measure the attenuation characteristics based on the objective method (without the presence of the subject), the results of which will be in accordance with the results of subjective tests. Therefore, the main purpose of the research in this work was to measure the attenuation characteristics of individual hearing protectors with acoustic filters through the use of subjective and objective methods, and to compare the results in terms of the research methods.
\end{abstract}

Methods: Measurements of the acoustic attenuation obtained by individual hearing protectors with designed F1, F2 and F3 acoustic filters, as well as full insert earplugs (without any acoustic filters) were carried out using two methods: objective and subjective. The objective measurements were carried out in an anechoic chamber. The artificial head (High-frequency Head and Torso Simulator Brül \& Kjær Type 5128) was located at a distance of $3 \mathrm{~m}$, directly opposite the loudspeaker. The test signal in the measurements was pink noise - in the frequency range up to $12.5 \mathrm{kHz}$ and the level 85, 90 and $95 \mathrm{~dB}$. The hearing protectors with and without acoustic filters were mounted in the Head and Torso Simulator which was connected with Pulse System Brül \& Kjær. Five normal hearing subjects participated in the subjective measurements. A pink noise signal was used for one-third octave bands: 125, 250, 500, 1000, 2000, 4000 and $8000 \mathrm{~Hz}$. The attenuation value was defined as the difference (in $\mathrm{dB}$ ) between the hearing threshold of the test signal with a hearing protector and the hearing threshold determined without a hearing protector.

Results: The results of the objective method proved that in addition to the significant impact of frequency on the attenuation values, the type of filter used also had a significant effect. The objective measurement method showed that different levels of stimulation of the test signal did not significantly affect the attenuation efficiency for 
both the full earplugs and the earplugs with the F1, F2 and F3 filters. In addition, the results of the objective method showed that in the whole frequency range the highest attenuation values are shown by the full earplugs, achieving slightly above $45 \mathrm{~dB}$ for frequency of $8 \mathrm{kHz}$. The attenuation values obtained from subjective measurements also confirmed that both the frequency and type of filter significantly affect the attenuation values of the tested hearing protectors. Unlike the results of the objective method, the subjective method did not indicate significant differences in attenuation when using $\mathrm{F} 1$ and $\mathrm{F} 2$ filters.

Conclusions: The comparison of the average attenuation values obtained from the objective and subjective methods showed that in general the measurement method does not significantly affect the average attenuation values. In turn, the analysis of variance broken down into subgroups according to the types of filters used in the earplugs showed that the influence of the measurement method on the attenuation values is statistically significant when the F1 filter and full earplug are used. The results of this study partly confirmed the hypothesis that there is no significant impact of the measurement method on the attenuation characteristics of the earplugs with different types of acoustic filters.

Keywords: hearing protector, earplug, acoustic filter, hearing loss, artificial head 


\section{Background}

Noise is defined as any unwanted sound which can be annoying or unsafe to human health, and which may increase the risk of an accident at work. Noise can cause sleep disturbance, worsening of speech intelligibility, as well as temporary or permanent hearing loss. Hearing loss is a process that usually develops over years, because it is usually painless, gradual and practically imperceptible. The progress of hearing loss due to the chronic influence of noise depends on the level of noise exposure and its duration. Hearing loss caused by excessive noise levels is one of the most common health risks for employees. Over 20\% of workers in Europe are at risk of permanent hearing loss. The safe and acceptable noise level, within an eighthour working day, is $85 \mathrm{~dB}$. This noise level is often exceeded: a comparable or higher noise level (instantaneous values) occurs for example, along highways. Noise at the workplace can be limited by both organizational methods and technical means. One solution for noise reduction is the use of hearing protectors, which is one of the most effective methods for protecting hearing from noise at the workplace. There are many types of hearing protectors: earmuffs, anti-noise earplugs and individual hearing protectors. Individual hearing protectors in the form of earplugs are made on the basis of the ear impression. Such earplugs, after insertion, tightly close the external auditory canal. In order to obtain different attenuation efficiency, individual hearing protectors can be equipped with a suitable acoustic filter. These are easily replaceable elements, which are selected depending on the acoustic conditions at the workplace. The selection of hearing protectors is carried out on the basis of measurements of sound pressure levels at the workplace. The choice of hearing protector means there is a need to determine the appropriate attenuation efficiency this should not allow excessive acoustic protection. Such excessive protection could make it impossible to communicate with other people and hear warning sounds.

In the available literature, research topics concerning hearing protectors are mainly focused on the problem of how protectors influence the ability to attenuate sounds with different frequency characteristics and changes in time (broadband noise, bands of noise, sound pulses, gunfire) (Davis et al., 2011; Biabani et al., 2017; Fackler et al., 2017; Samelli et al., 2018).

In addition, the effect of hearing protectors on the ability to determine the location of a sound source (Zimpfer and Sarafian, 2014; Brown et al., 2015; Lee and Casali, 2017), changes in the level of speech intelligibility (Bockstael et al., 2011; Norin et al., 
2011; Brown et al., 2015; Hiselius et al., 2015; Lee and Casali, 2017) as well as more complex aspects of sound sensations, e.g. changes in the perception of the sound of musical instruments by musicians using hearing protectors are also examined (Killion, 2012). It turns out that in the case of musicians, the sound levels can reach very high values, in the order of over $100 \mathrm{~dB}$, which, with long-term exposure, may also result in hearing loss.

One of the problems resulting from the use of hearing protectors is the deterioration of speech intelligibility, especially when speech is presented against the background of noise, and when the level of speech sounds is low. Active hearing protectors that enable the selection of signal enhancement depending on the sound level reaching the user are a partial solution here (Bockstael et al., 2011; Brown et al., 2015; Lee and Casali, 2017). However, in one paper (Norin et al., 2011), the authors showed that using 3 different passive hearing protectors did not determine the impact of the type of the protector on speech intelligibility against noise, and showed that none of the hearing protectors significantly affected speech intelligibility. The one significant factor influencing speech intelligibility was the signal-to-noise ratio (SNR).

In the case of active hearing protectors (signal amplification function depending on the level of the input signal) a significant improvement in speech intelligibility was found, especially for lower SNR values (Hiselius et al., 2015).

In the studies determining the effectiveness of the attenuation provided by hearing protectors, the key issue is the research methodology according to ISO 4869-1, the main advantage of which is real measurement of hearing thresholds for normalhearing people with and without hearing protectors.

Comparison of hearing thresholds allows the attenuation provided by the earplug for the respective frequency bands to be determined.

These attenuation values take into account all the subjective aspects of sound perception, but on the other hand, data collection is a time-consuming process and the obtained values are characterized by quite large inter-individual variability.

Therefore, the optimal solution is to measure the attenuation characteristics based on the objective method (without the presence of the subject), the results of which will be in accordance with the results of subjective tests.

The best solution is to use an artificial head (head simulator) that contains full copy of the auricles as well as the ear canal. There are many models of artificial heads with 
artificial ears currently on the market, however, this equipment does not have a full copy of the ear canal.

One of the most important works on the comparison of research methods to determine the value of the acoustic attenuation of hearing protectors is the paper (Berger, 2005). In this work, the author compared three methods of measuring the effectiveness of hearing protectors, i.e. the real-ear attenuation at threshold -REAT method - ISO 4869-1 standard and two objective methods: microphone in real earMIRE, consisting of placing the microphone in near the tympanic membrane, as well as the acoustical test fixtures - ATF, which uses artificial ear systems consisting of a microphone placed in a suitable cover connected with an acoustic coupler. The element of the artificial ear is connected to the amplifier system and the measuring system, which usually provides a comprehensive analysis of signals, mainly in terms of determining sound levels and assessing the spectral structure.

This work focuses on comparing the results obtained by REAT and ATF methods. The objective method (AFT) used in the research is based on the latest B\&K solution regarding the design solution of an artificial ear placed in an artificial head (Bruel \& Kjaer type 5128-C). The latest version of the artificial ear has a full mapping of the external auditory canal, which guarantees attachment of the ear and earplugs analogous to the real ear, and also allows for phenomena related to the occlusion effect and the influence of the residual volume of the ear canal on the transmission properties of acoustic energy. This technical solution is very important because the subject of the research in this work is individually fitted hearing protectors, placed partly in the external ear canal, ensuring the system is tightly closed and the possibility of using passive acoustic filters.

Therefore, the main purpose of the research in this work was to measure the attenuation characteristics of individual hearing protectors with acoustic filters through the use of subjective and objective methods, and to compare the results in terms of the research methods. It was assumed that the results of the objective and subjective studies would not show statistically significant differences. This will be the basis for further research and the development of objective and fast methods for determining the attenuation characteristics of individual hearing protectors

\subsection{Noise assessment at the workplace}

Due to the fact that hearing protectors are an extreme solution which protects the 
auditory system from the sounds of excessive levels, reference should be made to the requirements and standards applicable to the permissible noise levels in a specific work environment.

To assess noise at the workplace, the following quantities should be used (based on The Regulation of the Ministry of Labor and Social Policy, of 6 June 2014, on the maximum permissible concentrations and intensities of agents that are hazardous for health at the workplace (Laws, 2014):

- $L_{E X, 8 h}[\mathrm{~dB}]$ - the level of exposure to noise during an 8-hour day,

- $L_{E X, 8 h}[\mathrm{~dB}]$ - the level of exposure to noise during a 5-day working week.

The level of exposure to noise during an 8-hour day is defined as:

$$
L_{E X, 8}=L_{A e q, T_{e}}+10 \log \frac{T_{e}}{T_{o}},
$$

where $L_{A e q, T_{e}}$ is the time average sound level determined for the time of noise exposure, $T_{e}$, and $T_{o}=8$ hours $=28800 \mathrm{~s}$. $T_{e}$ is the time in which noise affects the hearing system.

The level of exposure to noise during a 5-day working week is defined by the following equation:

$$
L_{E X, W}=10 \log \left(\frac{1}{5} \sum_{i=1}^{n} 10^{0,1\left(L_{E X, 8 h}\right)_{i}}\right),
$$

where the index $i$ denotes the $i$-th day in a week, and $n=5$ - denotes the number of working days during the week.

In addition, according to the above regulation (The Regulation of the Ministry of Labor and Social Policy, of 6 June 2014, on the maximum permissible concentrations and intensities of agents that are hazardous for health at the workplace (Journal of Laws 2014, item 817), for the purpose of determining acoustic conditions at the workplace, additional quantities are used:

- $\quad L_{C \text {,peak }}[\mathrm{dB}]$ - the C-weighted peak sound pressure level,

- $L_{A, \max }[\mathrm{dB}]$ - the A-weighted maximum sound pressure level. 
In order to determine the noise level at the workplace, the following legal acts should be used:

- ISO 9612 Acoustics -- Determination of occupational noise exposure -Engineering method (PN-EN-ISO-9612)

- $\quad$ PN-N-01307 Noise. Permissible noise values in the workplace. Measurement requirements, (PN-N-01307, 1994)

- $\quad$-The Regulation of the Ministry of Labor and Social Policy, of 6 June 2014 (Laws, 2014).

The methods of measuring the quantities characterizing noise at workplaces have been presented in detail in (PN-N-01307, 1994). The standard also includes requirements for measuring the equipment, and the mode and frequency of measurements.

The standard presents two methods:

- the direct method, which is based on continuous measurement, during the time when the worker is exposed to noise, and on reading the values of determined quantities directly from meters, e.g. noise dosimeters or integrating sound level meters, and

- the indirect method, which involves measuring noise in a time shorter than the one being evaluated and applying appropriate mathematical relationships to determine the required quantities.

The permissible values at the workplace are defined in The Regulation of the Ministry of Labor and Social Policy, of 6 June 2014 (Laws, 2014). These values are presented in Tab. 1.

According to (The Regulation of the Ministry of Labor and Social Policy, of 6 June 2014, on the maximum permissible concentrations and intensities of agents that are hazardous for health at the workplace (Journal of Laws 2014, item 817) (Laws, 2014), in order to determine if there are no exceedances of the permissible noise levels at the workplace, individual values may not exceed the values given in the table above. If at least one of these values is not retained then it is stated that the 
workplace exceeds the permissible noise level and appropriate noise minimization measures should be taken.

When all the technical possibilities to reduce noise at the workplace are exhausted and one of the abovementioned values is still exceeded, the employer is obliged to provide the employees with hearing protection and inform them about the potential risk of hearing damage.

\subsection{Individual hearing protectors}

One way of providing hearing protection at the workplace is through the use of individual hearing protectors. Hearing protectors are earplugs made on the basis of impressions from the ears. They close the external auditory canal tightly, and can be equipped with additional, easily exchangeable acoustic filters, characterized by different values of acoustic attenuation. The selection of these filters depends on the acoustic conditions - i.e. on the noise level at the workplace. It should be remembered that the selection of a hearing protector with a specific acoustic filter should ensure an adequate level of communication and that warning sounds are audible. The method for estimating the sound level when hearing protection is used is described in the ISO 4869-2 standard (ISO 4869-2: 2018. Acoustics - Hearing protectors - Part 2: Estimation of Effective A-weighted sound pressure levels when hearing protectors are worn). A well-chosen hearing protector should ensure effective communication and hearing of warning sounds. A badly chosen hearing protector can lead to the effect of excessive protection. This effect is associated with too much noise suppression. This, in turn, may cause the employee to feel acoustic isolation from the surroundings, and to experience reduced communication possibilities and the lack of the ability to hear alarm and warning sounds. As a result, this leads to work discomfort and may lead to the employee's rejection of the hearing protector.

The construction of an individual hearing protector with an acoustic filter is presented in Figure 1. The acoustic filter is mounted in a special sleeve, which is permanently glued to the insert / protector.

To determine the acoustic efficiency (suppression) of individual hearing protectors, subjective and objective methods are used.

The subjective method for measuring the acoustic efficiency of hearing protectors is provided by (PN-EN-ISO-4869-3). This standard describes the subjective method of measuring the acoustic attenuation of hearing protectors at low sound pressure 
levels (close to the threshold of hearing). The method was developed to obtain attenuation values close to the maximum, which are difficult to achieve in real conditions. The hearing threshold is measured with and without a hearing protector (similar to (PN-EN-ISO-8253-2.)).

The objective method for measuring protectors is given in (PN-EN-ISO-4869-3). However, this standard applies to ear-muffs.

\section{Methods}

\subsection{The design of new acoustic filters}

New acoustic filters were designed and manufactured for the purposes of the research. The cross-section of one of these filters is shown in Figure 2. The three filters were designed with different diameters, $d$, of the inner hole. The filters were labelled with the symbols F1, F2 and F3, however the filter F1 had the smallest inner hole diameter, and F3 - the largest diameter. The larger the diameter of the inner hole, the smaller the sound reduction.

\subsection{The determination of the acoustics attenuation individual hearing protectors}

Measurements of the acoustic attenuation obtained by individual hearing protectors with designed F1, F2 and F3 acoustic filters, as well as full insert earplugs (without any acoustic filters) were carried out using two methods: objective (chapter 2.3) and subjective (chapter 2.4).

\subsection{The objective method - measurements using a head simulator}

The measurements were carried out in an anechoic chamber at the Institute of Acoustics of the Adam Mickiewicz University in Poznan.

The parameters of the anechoic chamber are presented Tab. 2.

The following measuring equipment was used in the measurements:

- High-frequency Head and Torso Simulator Brül\&Kjær Type 5128;

- Active Loudspeaker QSC type K10;

- System Pulse v. 12.6.0.255;

- Sound level meter Svantek type SVAN 945A;

The measurement system is presented in Figure 3. 
The acoustic measurements were made for the following conditions:

- without hearing protectors,

- with hearing protectors without acoustic filters (the so-called full insert) and with acoustic filters mounted in the insert with the internal holes having different diameters, F1, F2 and F3.

The test signal in the measurements was pink noise - in the frequency range up to $12.5 \mathrm{kHz}$. The signal was generated by a loudspeaker (Active Loudspeaker QSC type K10). The artificial head (High-frequency Head and Torso Simulator Brül \& Kjær Type 5128) was located at a distance of $3 \mathrm{~m}$, directly opposite the loudspeaker. In addition, a sound level meter was located above the artificial head to calibrate the measuring system.

The pink noise level was 85,90 and $95 \mathrm{~dB}$. The spectrum of the acoustic signal used in the tests is shown in Figure 4.

\subsection{The subjective method - measurements of the hearing thresholds}

The subjective acoustic measurements were carried out in accordance with ISO 4869-1. Five students - students of the Institute of Acoustics (aged 20 to 22) participated in the measurements. According to the standard [4], before starting the actual measurements, the subjects were checked for normal hearing. Hearing thresholds for participants should not exceed $15 \mathrm{~dB} \mathrm{HL}$ in the $0.125-2 \mathrm{kHz}$ band and $25 \mathrm{~dB} \mathrm{HL}$ for frequencies above $2 \mathrm{kHz}$. The test for each listener was repeated a minimum of 3 times for test signals, with the difference between the hearing thresholds for the middle frequencies not exceeding $6 \mathrm{~dB}$. Below in Figure 5 and Figure 6 are the audiograms of people participating in the study.

To determine the attenuation value obtained by of hearing protectors, a pink noise signal was used for one-third octave bands: 125, 250, 500, 1000, 2000, 4000 and $8000 \mathrm{~Hz}$.

The attenuation value was defined as the difference (in $\mathrm{dB}$ ) between the hearing threshold of the test signal with a hearing protector and the hearing threshold determined without a hearing protector.

Measurements were made using an Interacoustics AC40 audiometer. As part of the work, measurements for each subject (for earplugs with different filters) were repeated three times. The level of background noise in the testing room did not 
exceed the permissible values for background sound pressure levels presented in the standard.

\section{Results}

\subsection{The results of the objective method}

As stated earlier, acoustic measurements were made for three scenarios: without ear protectors, with protectors, and with acoustic filters. During the acoustic measurements, the hearing protectors were located on both ears of the artificial head. The measurements were repeated five times for each condition.

The acoustic efficiency (attenuation) of hearing protectors with and without acoustic filters was determined as the difference between the sound levels recorded without a hearing protector and with protectors - with different acoustic filters. Figure 7 shows the attenuation values determined for the protectors with filters F1, F2 and F3, and without a filter.

\subsubsection{Statistical analysis}

An ANOVA analysis of variance was used to perform the statistical analyses. The dependent variable was the attenuation value of the hearing protector, while the factors were: "frequency", "filter type" and "pink noise level". The overall analysis showed that "frequency" is a factor significantly affecting the attenuation values $\{F(6)$ $=4720.91, p<0.001\}$, similarly to "filter type" $\{F(3)=4225.49, p<0.001\}$. However, the level of stimulation of the used pink noise turned out to be a factor that did not significantly affect the values of the attenuation of the earplugs with acoustic filters and in the case of the full earplug $\{F(2)=0.841, p=0.432\}$.

The relationship between the average attenuation and the filter type for respective levels of stimulation is given in Figure 8.

The Tukey test showed that at the confidence level $p=0.05$, the average values of attenuation for earplugs with filters F1, F2, F3 and full earplugs (FE) differ significantly.

Figure 9 shows the average values of attenuation as a function of frequency for individual earplugs equipped with F1, F2 and F3 filters and for the full earplug. It can be unequivocally stated that in the whole frequency range the greatest attenuation is shown by the full earplug, achieving for $8 \mathrm{kHz}$ average attenuation slightly above 45 
$\mathrm{dB}$. In the $0.5-2 \mathrm{kHz}$ frequency band, the average attenuation increases by approx. $20 \mathrm{~dB}$ / octave, regardless of the type of earplug. In turn, in the case of earplugs with filters, the maximum attenuation falls on the $2-4 \mathrm{kHz}$ frequency band, and for $4-8 \mathrm{kHz}$ decreases by about $6 \mathrm{~dB}$.

Analysis of the results by groups, according to the types of earplugs (with filters, or full earplug) showed that for filters F1 and F2 the average attenuation is constant in the frequency band 250-500 $\mathrm{HZ}$, while for each of the other frequencies the attenuation value is different, and these differences are statistically significant.

In the case of the F3 filter, constant attenuation values were obtained in the 2000$4000 \mathrm{~Hz}$ band, while for the other attenuation frequencies they were statistically significantly different. On the other hand, in the case of the full earplug, uniform attenuation values were obtained in the $4-8 \mathrm{kHz}$ band.

\subsection{The results of the objective method}

The obtained values of acoustic attenuation for individual subjects and the average attenuation values for the full earplugs with F1 - F3 filters are shown in Figure 10 Figure 13. Figure 14 shows a comparison of the attenuation for individual earplugs with acoustic filters and for the full earplugs. Figure 15 shows the attenuation values averaged over all the frequencies for individual earplugs with F1, F2, F3 filters and for the full earplug.

\subsubsection{Statistical analysis}

As part of the study, an ANOVA analysis was performed, in which the dependent variable was "attenuation", and the factors were: "frequency", "filter type". The main analysis of variance showed that both factors were statistically significant, at the significance level $p \leq 0.001$. This means that both "frequency" $\{F(6)=111.41, p=$ $0.001\}$ and "filter type" $\{F(3)=427.77, p=0.001\}$ significantly affect the attenuation values of the tested hearing protectors. Tukey's test showed that at the confidence level $p=0.05$, the attenuation values for earplugs with F1 and F2 filters do not differ from each other, while there are significant differences in the attenuation values for the other cases, i.e. for the F3 filter and for full earplug (FE).

This means that for all types of earplugs with filters and the full earplug the value of attenuation changed as a function of frequency, however the average values of attenuation between F1 and F2 were not statistically significant. It can therefore be 
concluded that the attenuation characteristics of the earplugs with the F1 and F2 filters are comparable. Only the use of the F3 filter significantly changed the attenuation characteristics. Therefore, to attain greater diversity in the attenuation characteristics of the earplugs with filters, a filter with a diameter smaller than the F1 and F2 filters, and larger than the F3 filter, should be used. In addition, a filter with a smaller diameter than F3 should be used to obtain higher attenuation values, especially in the higher frequency band.

When analyzing the results by group, according to the types of filters, it turned out that for earplugs with the F1 and F2 filters, the average attenuation values increase with increasing frequency and differ significantly for the $0.125,1,2,4$ and $8 \mathrm{kHz}$ frequency bands. It is only in the $0.25-0.5 \mathrm{kHz}$ bandwidth that the attenuation values of the F1 and F2 filters do not show significant statistical differences. A similar relationship of attenuation values as a function of frequency was observed for the F3 filter. A comparison of the frequency response characteristics of earplugs with individual types of acoustic filters is given in Figure 16.

The largest differences of the frequency response attenuation in relation to the $F 1$, F2 and F3 filters are shown by the full earplug (FE), i.e. it shows less selectivity of attenuation in individual frequency bands. The highest attenuation value falls within the $2-8 \mathrm{kHz}$ frequency range and is approximately $36 \mathrm{~dB}$, while in the $0.125-1 \mathrm{kHz}$ range the attenuation value is smaller and amounts to approximately $24 \mathrm{~dB}$.

The above analysis shows that the earmold combined with a properly selected acoustic filter allows to obtain a large diversity of attenuation values in each of the analyzed frequency bands, which cannot be obtained with a full earplug.

\subsection{A comparison of the results obtained by the objective and subjective methods}

The next stage of the analysis consisted in comparing the obtained results of acoustic attenuation for a full earplugs and earplugs with different filters (F1-F3), as obtained by the subjective method and the objective method. Statistical analysis showed that for objective measurements the effect of the level of pink noise stimulation was not statistically significant, therefore this factor was not taken into account in these analyses. The factors were: "method", "frequency" and "filter type". A general analysis of ANOVA variance showed that "frequency" and "filter type" are factors that significantly affect insert values $\{F(6)=757.49, p<0.001\}$ and $\{F(3)=$ 
1340.46, $\mathrm{p}<0.001\}$. However, it was shown that the "method" turned out to be statistically insignificant $\{F(1)=0.36, p=0.547\}$.

Figure 17 shows the average attenuation values over all types of earplugs as a function of frequency for the objective and subjective methods.

Fig. 17 shows that the greatest differences in attenuation obtained by the subjective and objective methods occur for the frequencies of 0.25 and $4 \mathrm{kHz}$.

Further analyzes were carried out independently in four groups, depending on the type of filter used in the earplugs and also for the full earplug. The results obtained are shown in Figure 18 (for filter F1), Figure 19 (for filter F2), Figure 20 (for filter F3) and Figure 21 (for the full earplug).

The analysis of variance made with the abovementioned subgroups showed that the impact of the measurement method on the attenuation values is statistically significant when the F1 filter and full earplug are used.

The largest differences in attenuation values between the type of measurement methods occur for the low frequency band (up to $250 \mathrm{~Hz}$ ) and for high frequencies (above $4000 \mathrm{~Hz}$ ). Most likely, this is related to the transmission of acoustic energy through vibrations of the skull / artificial head bones. In the low frequency band, bone conduction plays an important role in stimulating the inner ear and can be a significant factor in lowering the hearing threshold in this frequency band. However, for high frequencies the attenuation of airborne sound in an artificial head may be less than that of a real ear.

\section{Discussion}

One of the most important papers on measuring the effectiveness of the attenuation caused by of hearing protectors is the publication (Berger, 2005). The author compared three methods of measuring the effectiveness of hearing protectors, i.e. the real-ear attenuation at threshold (REAT) method based on measuring the hearing threshold of people with hearing protectors, and two objective methods. The first objective method is analogous to that used in this work, based on a measuring system consisting of an artificial ear / artificial head (acoustical test fixtures - ATF). The second objective method is to measure the sound level in the ear canal after putting on the hearing protector by placing a microphone near the eardrum (microphone in real ear-MIRE). In the conclusion of the work (Berger, 2005), they stated that the REAT method is considered optimal, although it is not without 
drawbacks, such as those resulting from the need to involve a group of people to perform time-consuming measurements. When the MIRE method is used, bone conduction is not included in the process of acoustic energy transmission.

The optimal solution would be to use the ATF method to measure the attenuation characteristics of hearing protectors, both over-the-ear and in-the-ear, and this has been attempted in this study.

The work (Kozlowski and Mlynski, 2017) focused on determining the attenuation characteristics of ear protectors in the band above $8 \mathrm{kHz}$, i.e. $10,12.5$ and $16 \mathrm{kHz}$. Ear protectors (earmuffs) were used. The subjective (REAT) and objective methods were also used based on the "acoustic text fixture - ATX" - the artificial head of G.R.A.S. 45CB. The authors of this study examined a total of 27 hearing protectors (different companies and types) and obtained large differences in attenuation between individual types of protectors, reaching up to $40 \mathrm{~dB}$ at $16 \mathrm{kHz}$. In addition, they found differences between the attenuation results based on different methods. For the $10 \mathrm{kHz}$ frequency, the average attenuation values determined by the objective method were higher by $6 \mathrm{~dB}$, while for the 12.5 and $16 \mathrm{kHz}$ frequencies they were greater and these differences on average even amounted to $15 \mathrm{~dB}$.

(Chordekar et al., 2016) investigated the effect of factors limiting the attenuation values for earplugs and earmuffs. They assumed that hearing loss, occlusion or bone conduction might be responsible for reducing the attenuation of hearing protectors. They tested the effectiveness of attenuation provided by hearing protectors using the subjective REAT method. Measurements were made for earmuffs, earplugs and simultaneously used earplugs and earmuffs. The test results showed that the use of ear protectors with the earplugs in place does not change the attenuation efficiency. They showed that the transmission reduction of vibrations through soft tissues is responsible for influencing a reduction in the attenuation provided by hearing protectors, also in the case of earplugs placed deep in the ear canal.

(Wulf-Andresen and Rasmussen, 2015) measured the attenuation of passive and active hearing protectors based on an objective method, i.e. using the artificial head AFT 45CB and AFT (45CA). They examined three different types of passive hearing protectors (circum-aural, soft flange in ear and simple foam in-ear) and three types of active hearing protectors (supra-aural, circum-aural and one in-ear canal). They stated that the test results do not depend on the devices used (AFT 45 CB and AFT $45 \mathrm{CA})$. 
(Mlynski et al., 2014) studied the effectiveness of attenuation of two types of hearing protectors in the case of impulse noise, which was the impact of a steel hammer at the workplace. In research, they used the objective AFT method. Test results have shown that the highest attenuation efficiency was demonstrated by earplugs.

The article (Bockstael et al., 2008) is an interesting work on the use of hearing protectors in the case of noise from firearms. The authors also measured two types of otoacoustic emissions (distortion product otoacoustic emission - DPOAE, and transiently-evoked otoacoustic emission -TEOAE) in soldiers who used hearing protectors while shooting. The subjects were divided into two groups, one group used passive earplugs, the other one used active earmuffs as hearing protectors. DPOAE and TEOAE measurements were taken before and immediately after shooting for a period of 5 days. The test results showed that, regardless of the type of hearing protectors, no significant changes in OAE levels were found after exposure to noise from firearms. This indicates that the inner ear cochlea is sufficiently protected against noise at too high a sound pressure level.

\section{Conclusions}

To sum up, the most important conclusions resulting from this work are as follows:

- In the case of an objective method of measuring the attenuation provided by earplugs, it was shown that in addition to the significant effect of frequency $\{F$ (6) $=4720.91, p<0.001\}$, the attenuation values also significantly depended on the type of acoustic filter used $\{F(3)=4225.49, p<0.001\}$.

- The objective measurement method showed that different levels of stimulation $(85,90$ and $95 \mathrm{~dB})$ of the test signal, which was pink noise, did not significantly affect the attenuation efficiency for either the full earplug and for the earplugs with F1, F2 and F3 filters.

- Tukey's test showed that at the confidence level $p=0.05$, the average attenuation values for earplugs with F1, F2, F3 filters and full earplugs obtained from objective measurements differ significantly from each other.

- The results of the objective method indicated that in the whole frequency range the highest attenuation is shown by the full earplugs, achieving $8 \mathrm{kHz}$ average attenuation slightly above $45 \mathrm{~dB}$. In turn, in the case of earplugs with filters, the maximum attenuation falls in the $2-4 \mathrm{kHz}$ frequency band, and for 4$8 \mathrm{kHz}$ decreases by about $6 \mathrm{~dB}$. 
- The attenuation values obtained from subjective measurements also confirmed that both the frequency $\{F(6)=111.41, p=0.001\}$ and the type of filter $\{F(3)=427.77, p=0.001\}$ significantly affect the attenuation values of the tested hearing protectors.

- In contrast to the results of the objective method, the subjective method did not indicate significant differences in attenuation when using the F1 and F2 filters.

- The comparison of the average attenuation values obtained from the objective and subjective methods showed that overall the measurement method does not significantly affect the mean attenuation values $\{F(1)=0.36, p=0.547\}$. In turn, analysis of variance broken down into subgroups due to the types of filters used in the earplugs showed that the impact of the measurement method on the attenuation values is statistically significant when the F1 filter and full earplug are used.

- The results of this study partly confirmed the hypothesis that there is no significant impact of the measurement method on the attenuation characteristics of the earplugs with different types of acoustic filters.

It should be added that the increasingly better systems of artificial ear / artificial head, especially those in which the full projection of the ear canal has been used, gives reason to hope that in the future fully objective measurement of the attenuation provided by any hearing protectors and earplugs will be possible. The research results included in this work are preliminary studies. The plan is to use earplugs with a greater variety of acoustic filters in subsequent research and to significantly increase the group of people surveyed with the subjective method.

\section{List of Abbreviations}

SNR - signal-to-noise ratio

REAT - real-ear attenuation at threshold

ATF - acoustical test fixture

ANOVA - analysis of variance

FE - full earplug

MIRE - microphone in real ear

DPOAE - distortion product otoacoustic emission 
TEOAE - transiently-evoked otoacoustic emission

\section{Ethics approval and consent to participate}

The research was carried out in accordance with the recommendations of conducting scientific and didactic research with the participation of people specified in the Resolution of the Council of the Faculty of Physics of the University of Adam Mickiewicz in Poznan from December 20, 2013. All subjects agreed to participate in the study.

\section{Consent for publication}

All of the authors have approved the contents of this paper and have agreed to the Archives of Public Health's submission policies.

\section{Availability of data and material}

Not applicable.

\section{Competing interests}

The authors have no conflict of interest, financial or otherwise.

\section{Funding}

Not applicable.

\section{Authors' contributions}

RG: Contributions to the conception and the design of the study, data acquisition, analysis, results interpretation and writing the manuscript. AW: Contributions to the conception and the design of the study, analysis, results interpretation and writing the manuscript. AD: Design and implementation of earplugs MKK: data acquisition $\mathrm{KMH}$ : data acquisition.

\section{Acknowledgements}

We would like to thank dr Mikołaj Baranowski for developing and printing acoustic filters for the earplugs and subjects for participating in the studies. 


\section{References}

Berger, E. H. (2005). "Preferred Methods for Measuring Hearing Protector Attenuation," in Inter-Noise (Inter-Noise, 07-10 August, Rio de Janeiro).

Biabani, A., Aliabadi, M., Golmohammadi, R., and Farhadian, M. (2017). "Individual Fit Testing of Hearing Protection Devices Based on Microphone in Real Ear," Saf Health Work 8, 364-370.

Bockstael, A., De Coensel, B., Botteldooren, D., D'Haenens, W., Keppler, H., Maes, L., Philips, B., Swinnen, F., and Bart, V. (2011). "Speech recognition in noise with active and passive hearing protectors: a comparative study," J Acoust Soc Am 129, 3702-3715.

Bockstael, A., Keppler, H., Dhooge, I., D'haenens, W., Maes, L., Philips, B., and Vinck, B. (2008). "Effectiveness of hearing protector devices in impulse noise verified with transiently evoked and distortion product otoacoustic emissions," Int J Audiol 47, 119-133.

Brown, A. D., Beemer, B. T., Greene, N. T., Argo, T. t., Meegan, G. D., and Tollin, D. J. (2015). "Effects of Active and Passive Hearing Protection Devices on Sound Source Localization, Speech Recognition, and Tone Detection," PLoS One 10, e0136568.

Chordekar, S., Adelman, C., Sohmer, H., and Kishon-Rabin, L. (2016). "Soft Tissue Conduction as a Possible Contributor to the Limited Attenuation Provided by Hearing Protection Devices," Noise Health 18, 274-279.

Davis, R. R., Murphy, W. J., Byrne, D. C., and Shaw, P. B. (2011). "Acceptance of a semi-custom hearing protector by manufacturing workers," J Occup Environ Hyg 8, D125-130.

Fackler, C. J., Berger, E. H., Murphy, W. J., and Stergar, M. E. (2017). "Spectral analysis of hearing protector impulsive insertion loss," Int J Audiol 56, 13-21.

Hiselius, P., Edvall, N., and Reimers, D. (2015). "To measure the impact of hearing protectors on the perception of speech in noise," Int J Audiol 54, S3-S8.

Killion, M. C. (2012). "Factors Influencing Use of Hearing Protection by Trumpet Players," Trends Amplif 16, 173-178.

Kozlowski, E., and Mlynski, R. (2017). "Measurement of Earmuffs Attenuation at High Audible Frequencies," Arch Acoust 42, 249-254. 
Laws, J. o. (2014). "The Regulation of the Ministry of Labor and Social Policy, of 6 June 2014, item 817, on the maximum permissible concentrations and intensities of agents that are hazardous for health at the workplace ".

Lee, K., and Casali, J. G. (2017). "Development of an auditory situation awareness test battery for advanced hearing protectors and TCAPS: detection subtest of DRILCOM (detection-recognition/identification-localization-communication)," Int J Audiol 56, 22-33.

Mlynski, R., Kozlowski, E., and Adamczyk, J. (2014). "Assessment of Impulse Noise Hazard and the Use of Hearing Protection Devices in Workplaces where Forging Hammers are Used," Arch Acoust 39, 73-79.

Norin, J. A., Emanuel, D. C., and Letowski, T. R. (2011). "Speech Intelligibility and Passive, Level-Dependent Earplugs," Ear Hearing 32, 642-649.

PN-EN-ISO-4869-3 (2007). "Acoustics. Hearing protectors -- Part 3: Measurement of insertion loss of ear-muff type protectors using an acoustic test fixture," (Polish Committee for Standardization).

PN-EN-ISO-8253-2. (2010). "Acoustics. Audiometric test methods -- Part 2: Sound field audiometry with pure-tone and narrow-band test signals," (Polish Committee for Standardization).

PN-EN-ISO-9612 (2011). "Acoustics. Determination of occupational noise exposure - Engineering method," in (Polish Committee for Standardization).

PN-N-01307 (1994). "Permissible noise values in the workplace. Measurement requirements," (Polish Committee for Standardization).

Samelli, A. G., Gomes, R. F., Chammas, T. V., Silva, B. G., Moreira, R. R., and Fiorini, A. C. (2018). "The Study of Attenuation Levels and the Comfort of Earplugs," Noise Health 20, 112-119.

Wulf-Andresen, P., and Rasmussen, P. (2015). "Standardized acoustic test fixtures for testing ear protection devices and noise cancelling headsets," in Euro Noise (EAA-NAG-ABAV, 31 May-3 June, Maastricht), pp. 1985-1990.

Zimpfer, V., and Sarafian, D. (2014). "Impact of hearing protection devices on sound localization performance," Front Neurosci-Switz 8. 
Tab. 1.The permissible values of noise at the workplace

\begin{tabular}{|l|c|}
\hline \multicolumn{1}{|c|}{ Noise descriptor } & $\begin{array}{c}\text { The permissible } \\
\text { value [dB] }\end{array}$ \\
\hline $\begin{array}{l}\text { Daily noise exposure level during an 8-hour day, } L_{E X, 8 h} \text { and } \\
\text { weekly noise exposure during a 5-day working week, } L_{E X, w}[\mathrm{~dB}]\end{array}$ & 85 \\
\hline The A-weighted maximum sound pressure level, $L_{A \max }[\mathrm{dB}]$ & 115 \\
\hline The C-weighted peak sound pressure level, $L_{C \text { Ceak }}[\mathrm{dB}]$ & 135 \\
\hline
\end{tabular}

Tab. 2. The parameters of the anechoic chamber Tab. 2 .

\begin{tabular}{|c|c|}
\hline Parameter & Value \\
\hline The dimensions (height / width / depth) $[\mathrm{m}]$ & $6.5 / 6.5 / 8.5$ \\
\hline The volume $\left[\mathrm{m}^{3}\right]$ & 360 \\
\hline The lower frequency limit $80[\mathrm{~Hz}]$ & 80 \\
\hline
\end{tabular}




\section{Figure captions}

Figure 1. Construction of an individual hearing protector (earplug). The left part of the Figure - the acoustic filter (red colour) is mounted in an acoustic tube (blue colour), which is permanently glued to the earplug. The right part of the Figure - an acoustic filter of white color and a bush in blue

Figure 2. Acoustic filter cross-section and longitudinal section

Figure 3. An artificial head with a torso made by the B\&K Company and a sound source placed opposite the dummy placed in an anechoic chamber. Front view (a) and side view of the head and torso simulator

Figure 4. One-third octave pink noise spectrum used in the study for three different total sound pressure levels ( $\mathrm{L} p=85,90$ and $95 \mathrm{~dB} S \mathrm{SPL})$

Figure 5. Audiograms of subjects participating in the study (results for the right ear).

Figure 6. Audiograms of subjects participating in the study (results for the left ear).

Figure 7. Mean attenuation values for hearing protectors (earplugs) with particular types of acoustic filters (F1, F2 and F3). The full earplug without filter was marked as $(\mathrm{FE})$. Error bars show \pm 1 standard deviation

Figure 8. Mean values of acoustic attenuation for the earplug with various acoustic filters (F1, F2 and F3) and for the full earplug (FE). Error bars show \pm 1 standard deviation

Figure 9. Dependence of mean sound attenuation as a function of frequency, for the earplug with different filters (F1, F2 and F3) and for the full earplug (FE). Error bars show \pm 1 standard deviation

Figure 10. Attenuation values for full earplug. Results for individual subjects and average values. Results obtained using the subjective method. Error bars show \pm 1 standard deviation

Figure 11. Attenuation values for earplug with acoustic filter F1. Results for individual subjects and average values. Results obtained using the subjective method. Error bars show \pm 1 standard deviation 
Figure 12. Attenuation values for earplug with acoustic filter F2. Results for individual subjects and average values. Results obtained using the subjective method. Error bars show \pm 1 standard deviation

Figure 13. Attenuation values for earplug with acoustic filter F3. Results for individual subjects and average values. Results obtained using the subjective method. Error bars show \pm 1 standard deviation

Figure 14. Mean values of attenuation as a function of frequency for earplugs with F1, F2 and F3 filter and for full earplug. Results obtained using the subjective method. Error bars show \pm 1 standard deviation

Figure 15. Mean values of attenuation for individual earplugs with acoustic filters (F1, F2 and F3) and for the full earplug (FE). Results obtained using the subjective method. Error bars show $95 \%$ confidence interval

Figure 16. Mean values of attenuation as a function of frequency for earplugs with F1, F2 and F3 filter and for full earplug. Results obtained using the subjective method. Error bars show $95 \%$ confidence interval

Figure 17. Mean values of attenuation depending on the frequency obtained with the objective method (empty squares) and the subjective method (empty circles). Error bars show a 95\% confidence interval

Figure 18. Mean values of attenuation for the earplug with filter F1 depending on the frequency obtained in the objective method (empty squares) and the subjective method (empty circles). Error bars show a $95 \%$ confidence interval

Figure 19. Mean values of attenuation for the earplug with filter F2 depending on the frequency obtained in the objective method (empty squares) and the subjective method (empty circles). Error bars show a 95\% confidence interval

Figure 20. Mean values of attenuation for the earplug with filter F3 depending on the frequency obtained in the objective method (empty squares) and the subjective method (empty circles). Error bars show a 95\% confidence interval 
Figure 21. Mean values of attenuation for the full earplug depending on the frequency obtained in the objective method (empty squares) and the subjective method (empty circles). Error bars show a 95\% confidence interval 
Figures

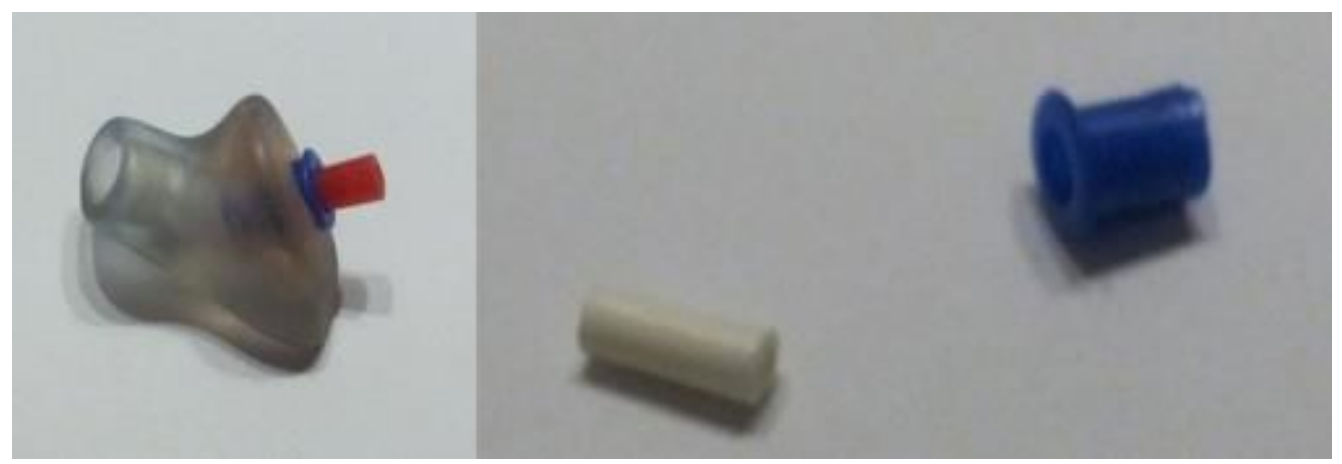

Figure 1

Construction of an individual hearing protector (earplug). The left part of the Figure - the acoustic filter (red colour) is mounted in an acoustic tube (blue colour), which is permanently glued to the earplug. The right part of the Figure - an acoustic filter of white color and a bush in blue
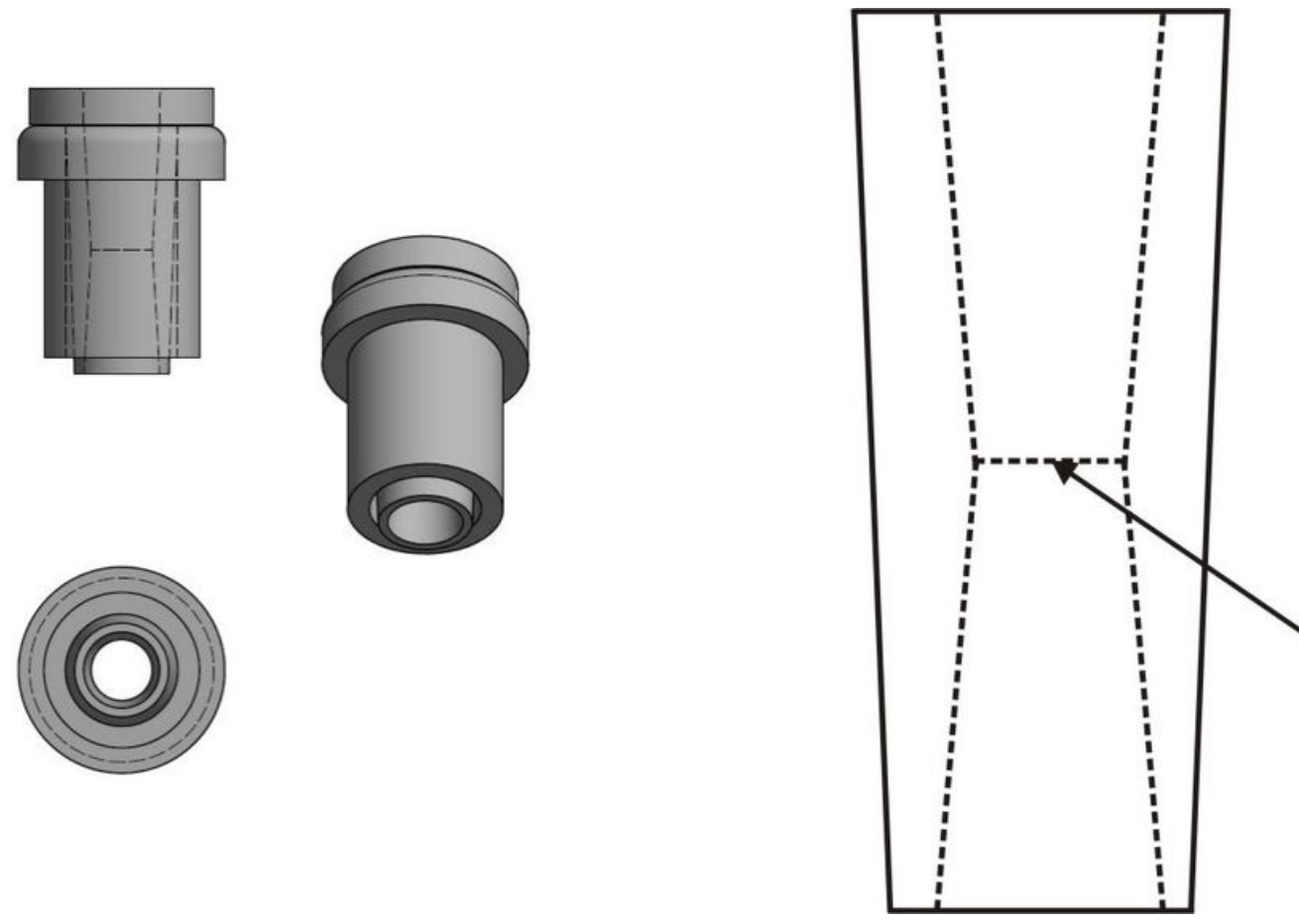

The diameter of the inner hole, $d$

\section{Figure 2}

Acoustic filter cross-section and longitudinal section 


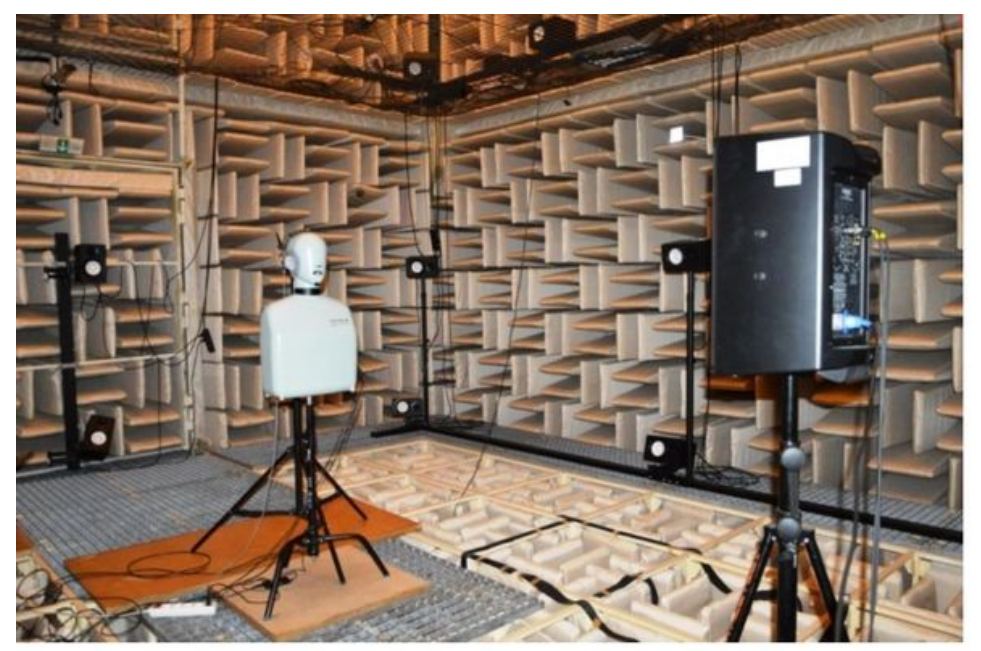

a)

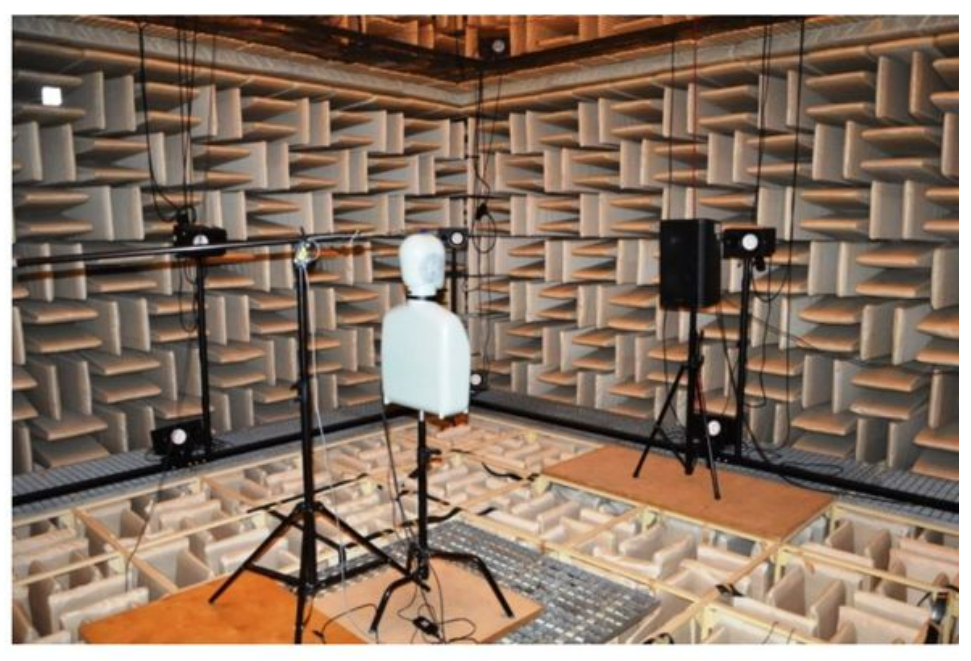

b)

Figure 3

An artificial head with a torso made by the B\&K Company and a sound source placed opposite the dummy placed in an anechoic chamber. Front view (a) and side view of the head and torso simulator

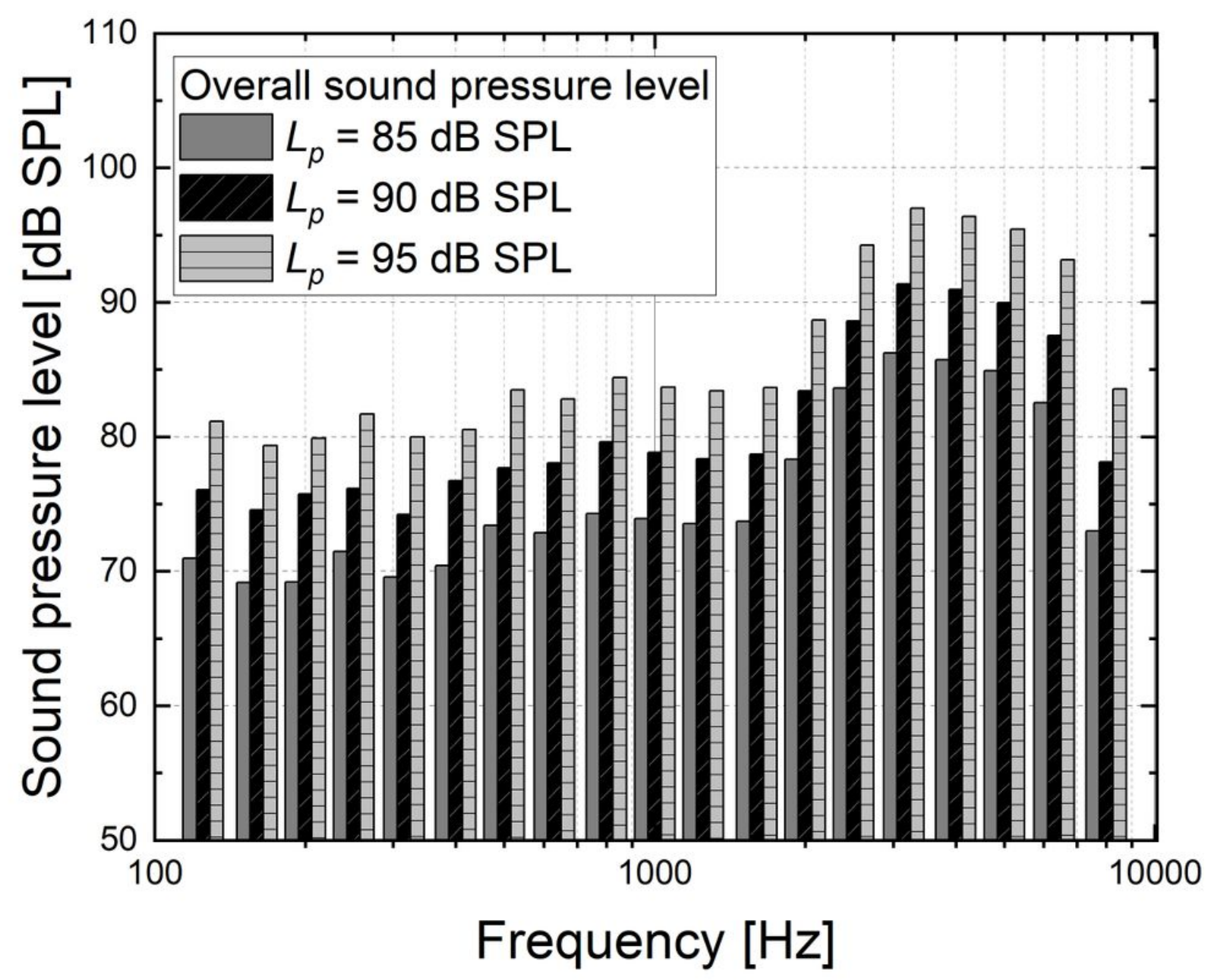


Figure 4

One-third octave pink noise spectrum used in the study for three different total sound pressure levels (Lp $=85,90$ and $95 \mathrm{~dB} \mathrm{SPL}$ )

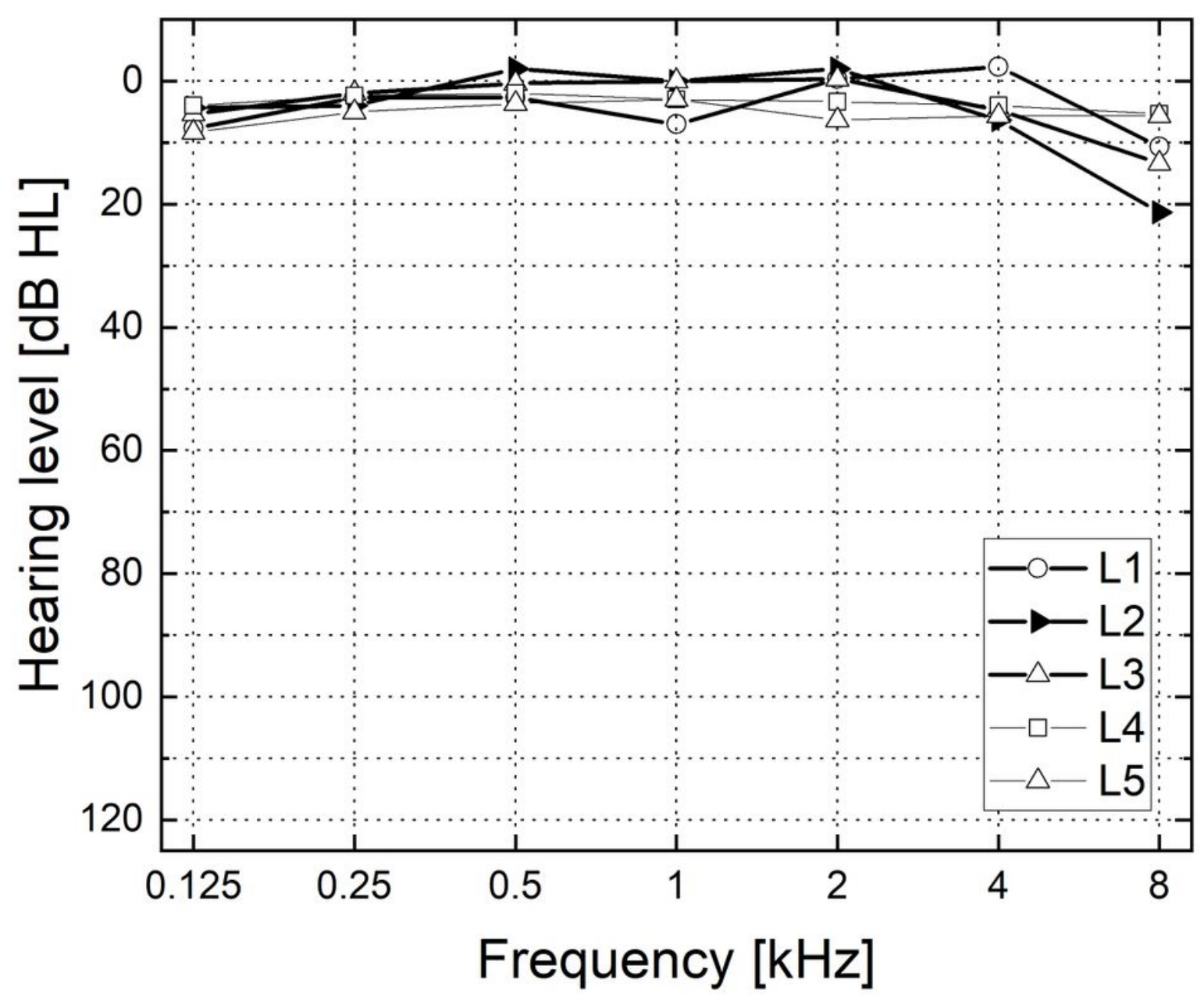

Figure 5

Audiograms of subjects participating in the study (results for the right ear). 


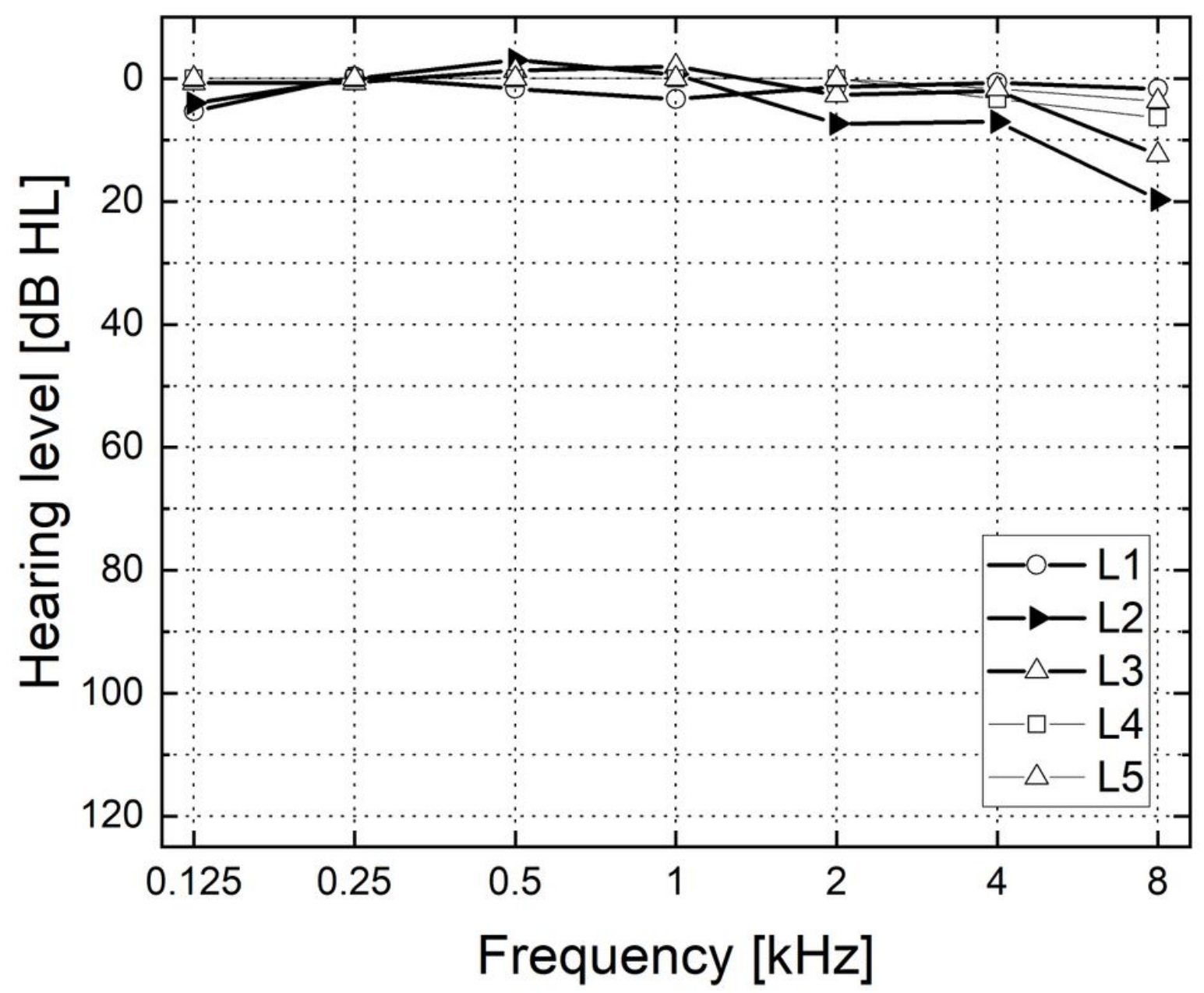

Figure 6

Audiograms of subjects participating in the study (results for the left ear). 


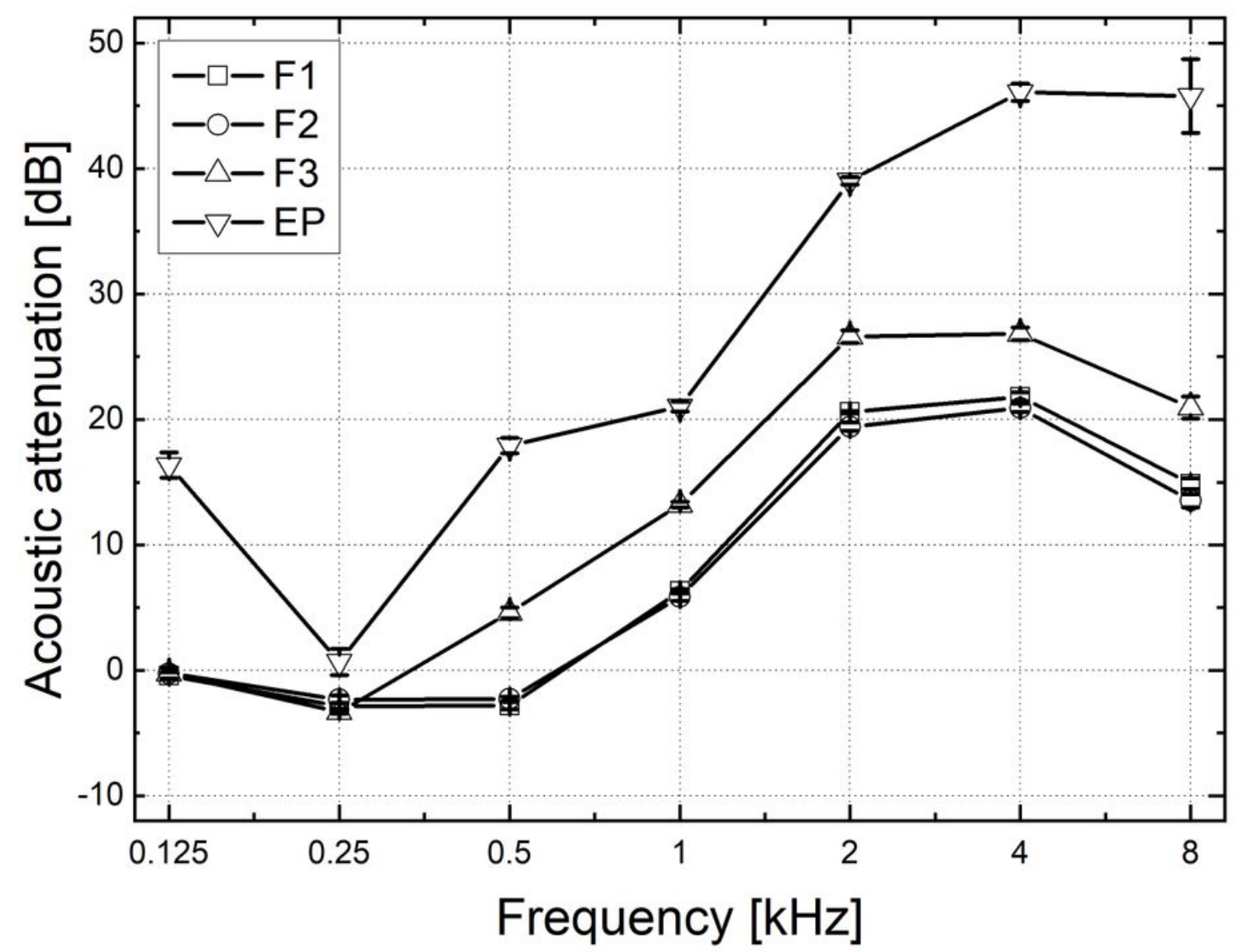

Figure 7

Mean attenuation values for hearing protectors (earplugs) with particular types of acoustic filters (F1, F2 and F3). The full earplug without filter was marked as (FE). Error bars show \pm 1 standard deviation 


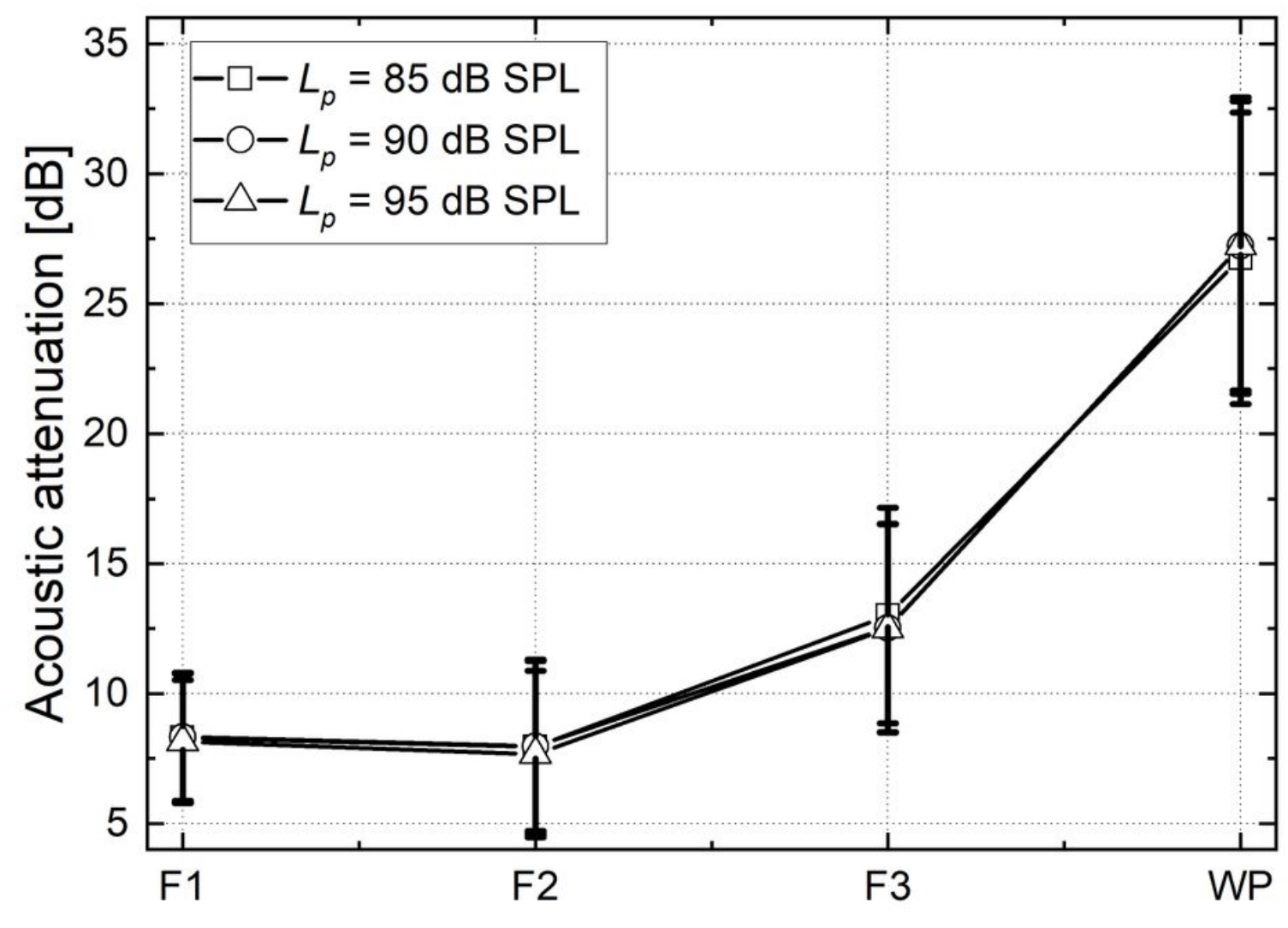

Filter type

Figure 8

Mean values of acoustic attenuation for the earplug with various acoustic filters (F1, F2 and F3) and for the full earplug (FE). Error bars show \pm 1 standard deviation 


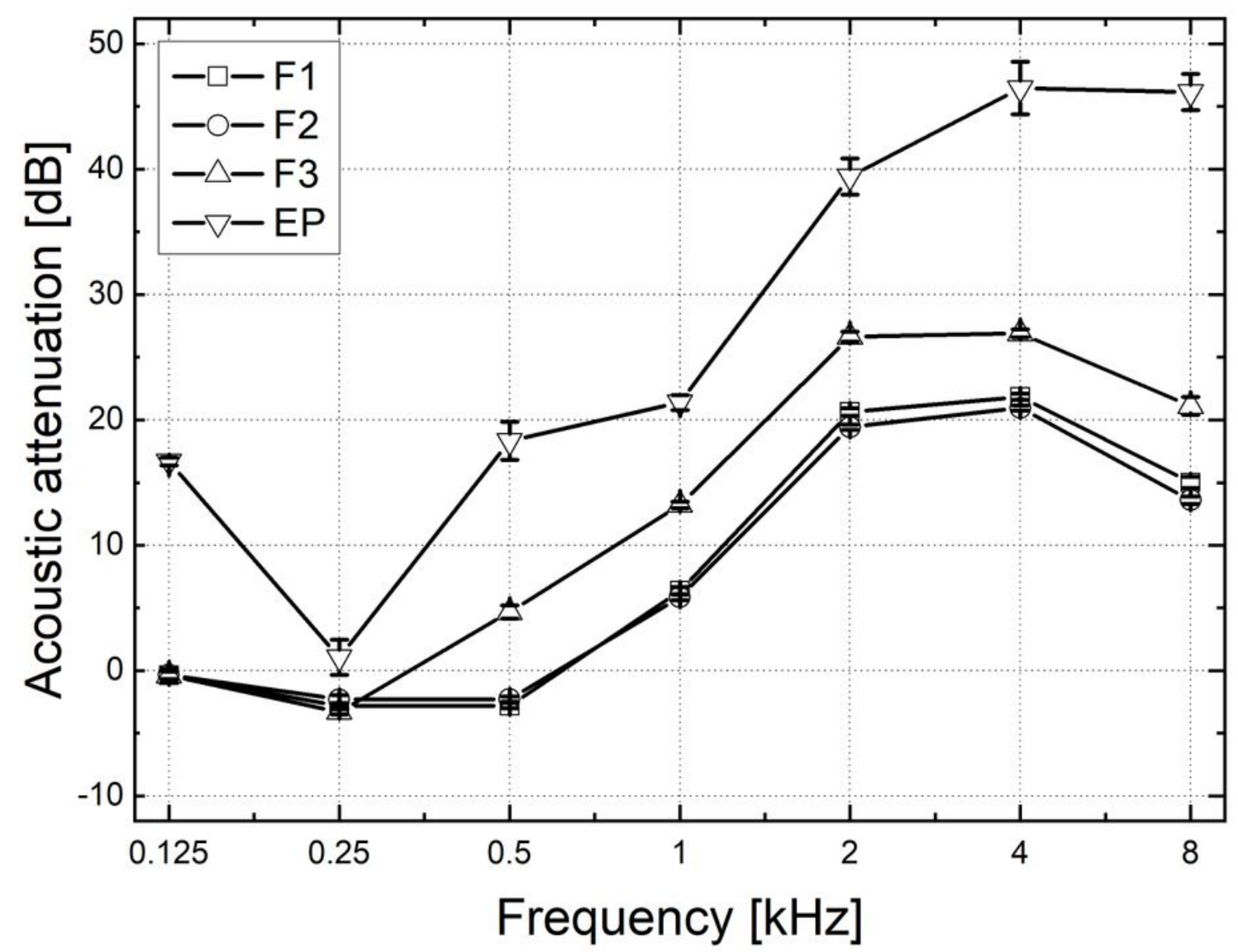

Figure 9

Dependence of mean sound attenuation as a function of frequency, for the earplug with different filters (F1, F2 and F3) and for the full earplug (FE). Error bars show \pm 1 standard deviation 


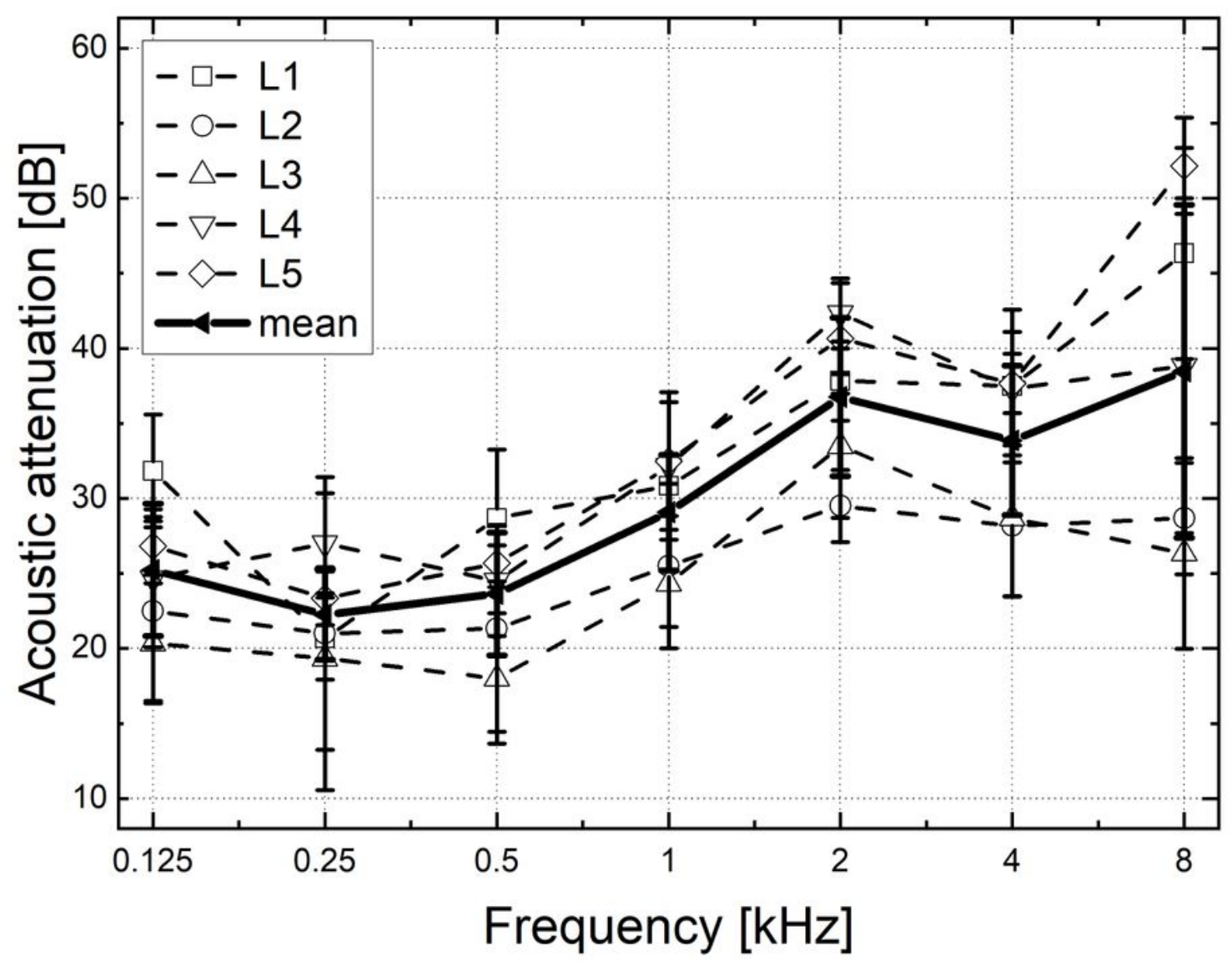

Figure 10

Attenuation values for full earplug. Results for individual subjects and average values. Results obtained using the subjective method. Error bars show \pm 1 standard deviation 


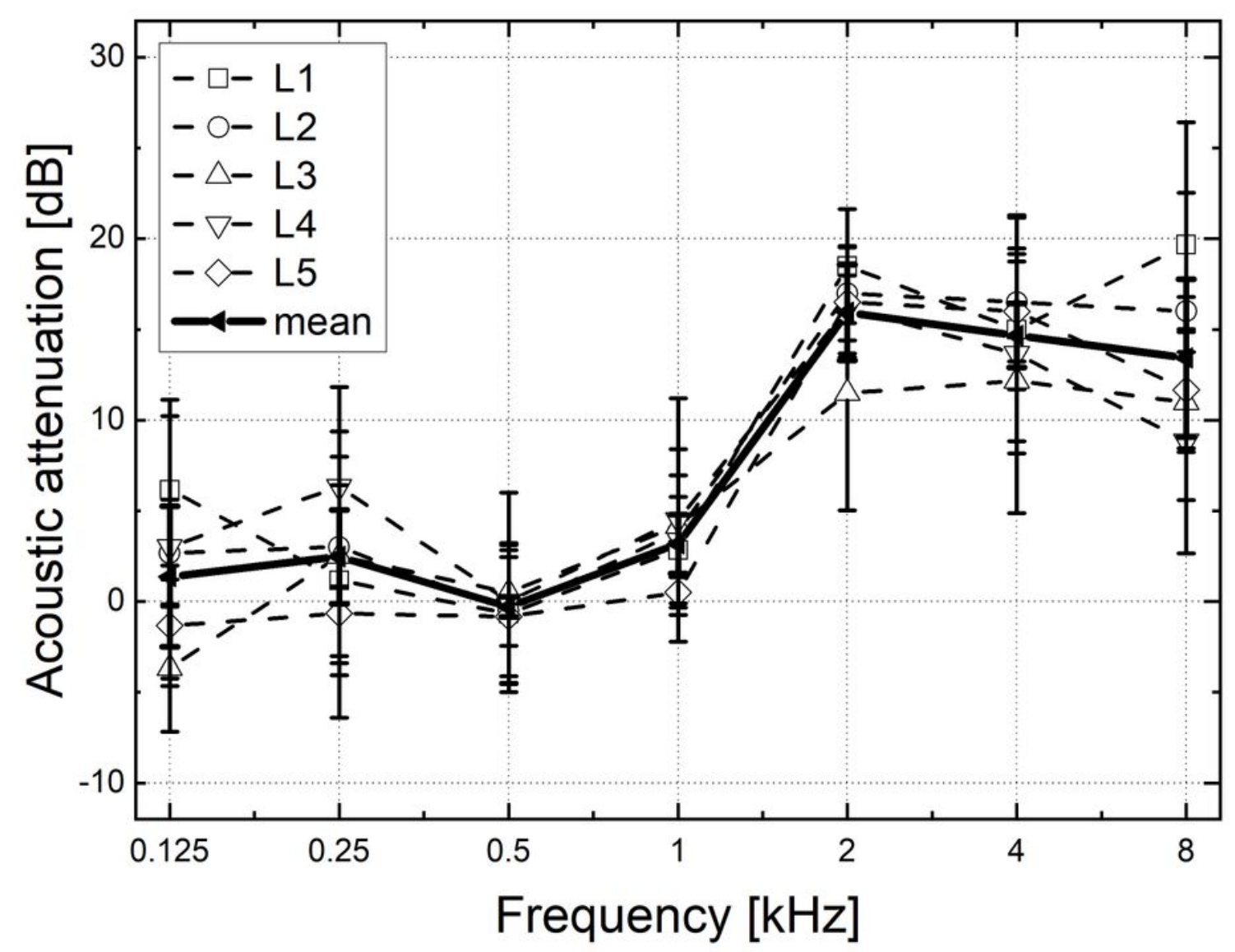

Figure 11

Attenuation values for earplug with acoustic filter F1. Results for individual subjects and average values. Results obtained using the subjective method. Error bars show \pm 1 standard deviation 


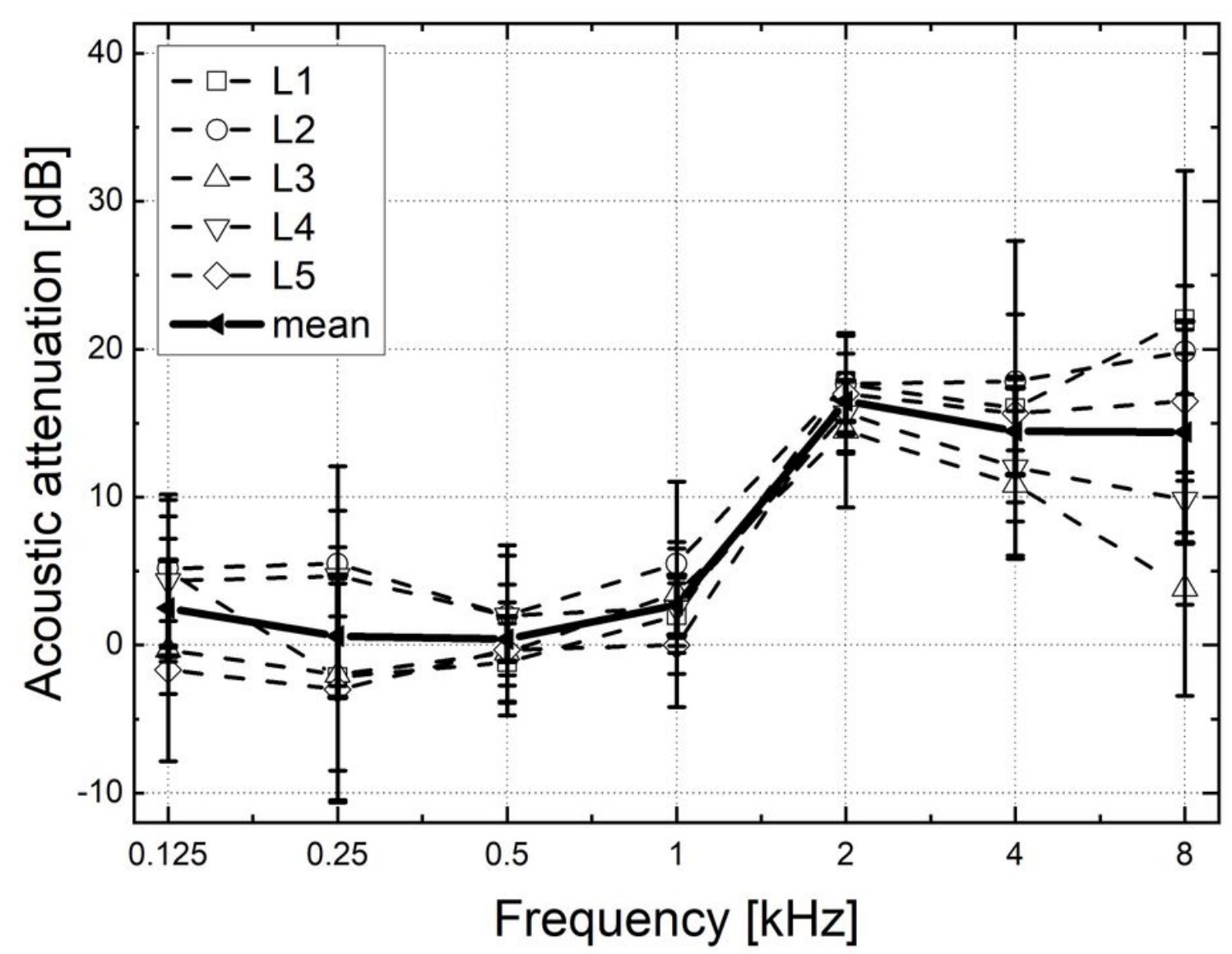

Figure 12

Attenuation values for earplug with acoustic filter F2. Results for individual subjects and average values. Results obtained using the subjective method. Error bars show \pm 1 standard deviation 


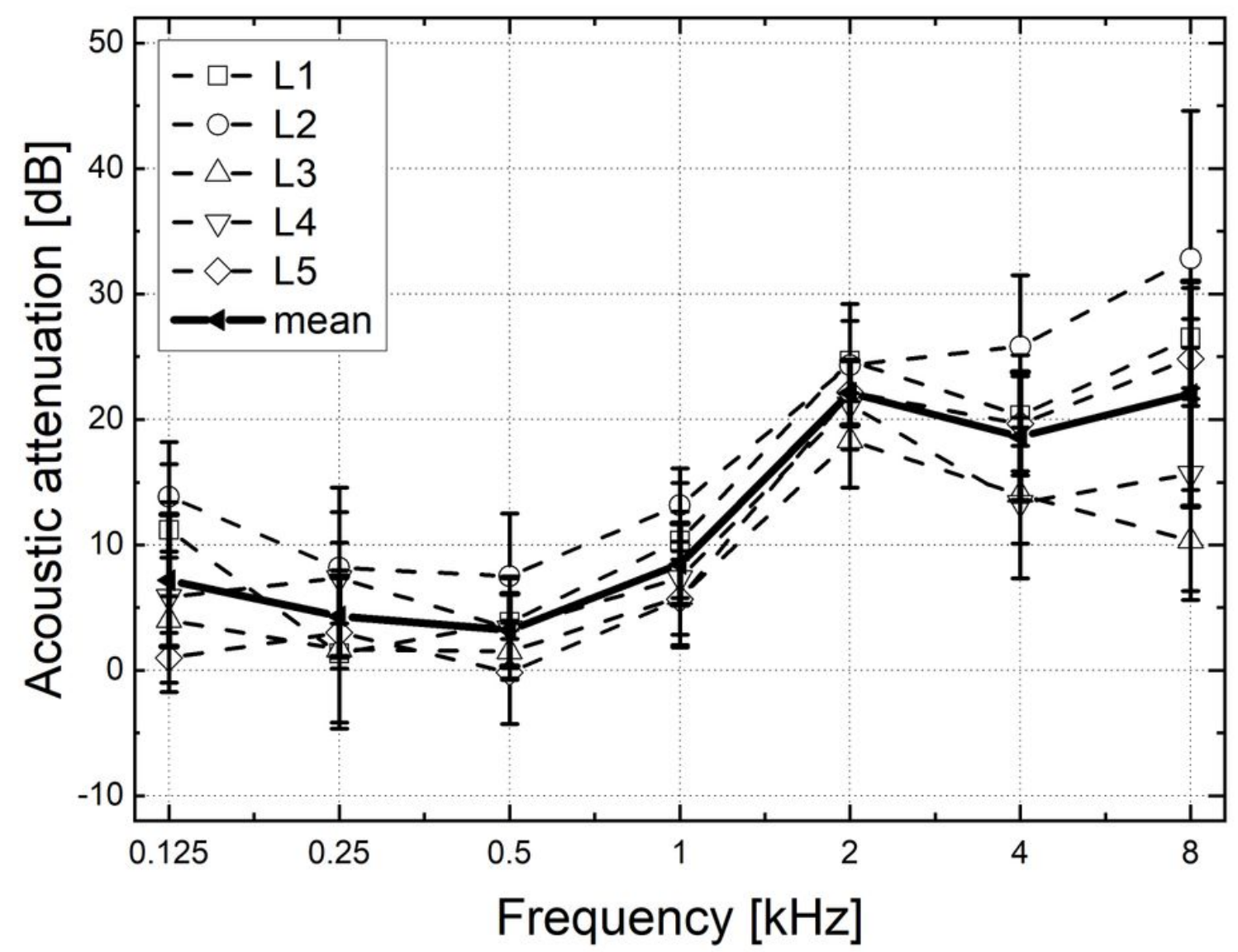

Figure 13

Attenuation values for earplug with acoustic filter F3. Results for individual subjects and average values. Results obtained using the subjective method. Error bars show \pm 1 standard deviation 


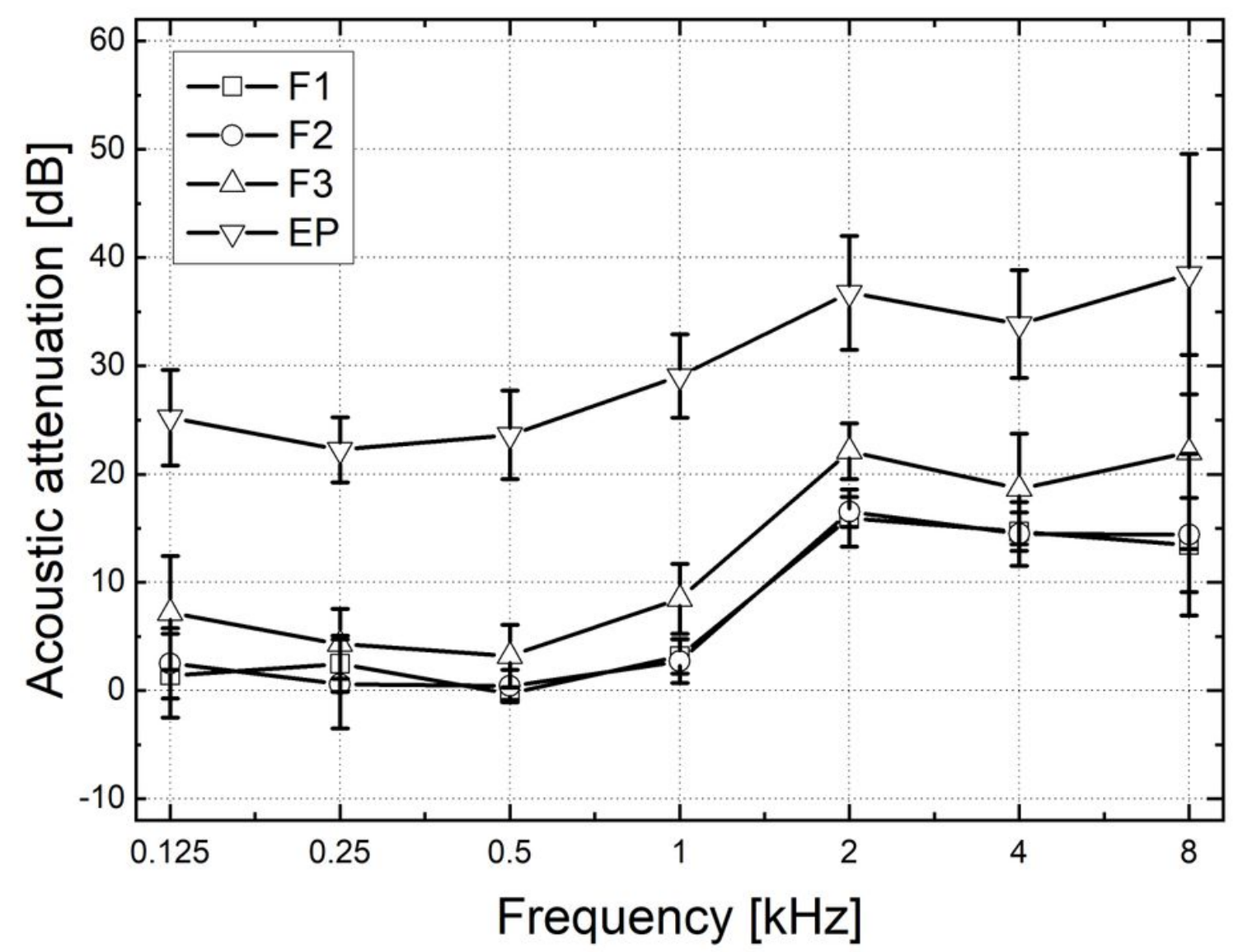

Figure 14

Mean values of attenuation as a function of frequency for earplugs with F1, F2 and F3 filter and for full earplug. Results obtained using the subjective method. Error bars show \pm 1 standard deviation 


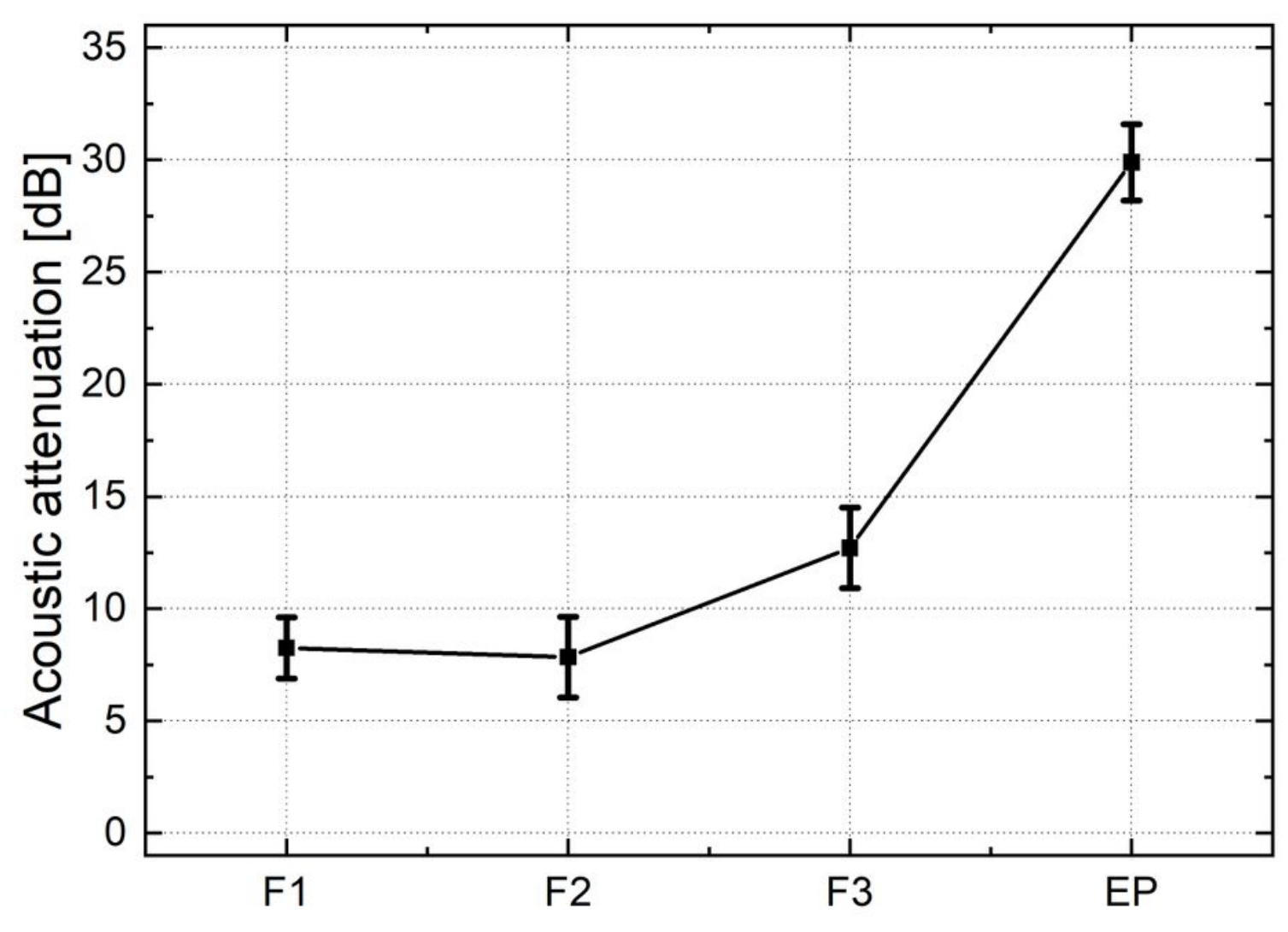

Filter type

Figure 15

Mean values of attenuation for individual earplugs with acoustic filters (F1, F2 and F3) and for the full earplug (FE). Results obtained using the subjective method. Error bars show $95 \%$ confidence interval 


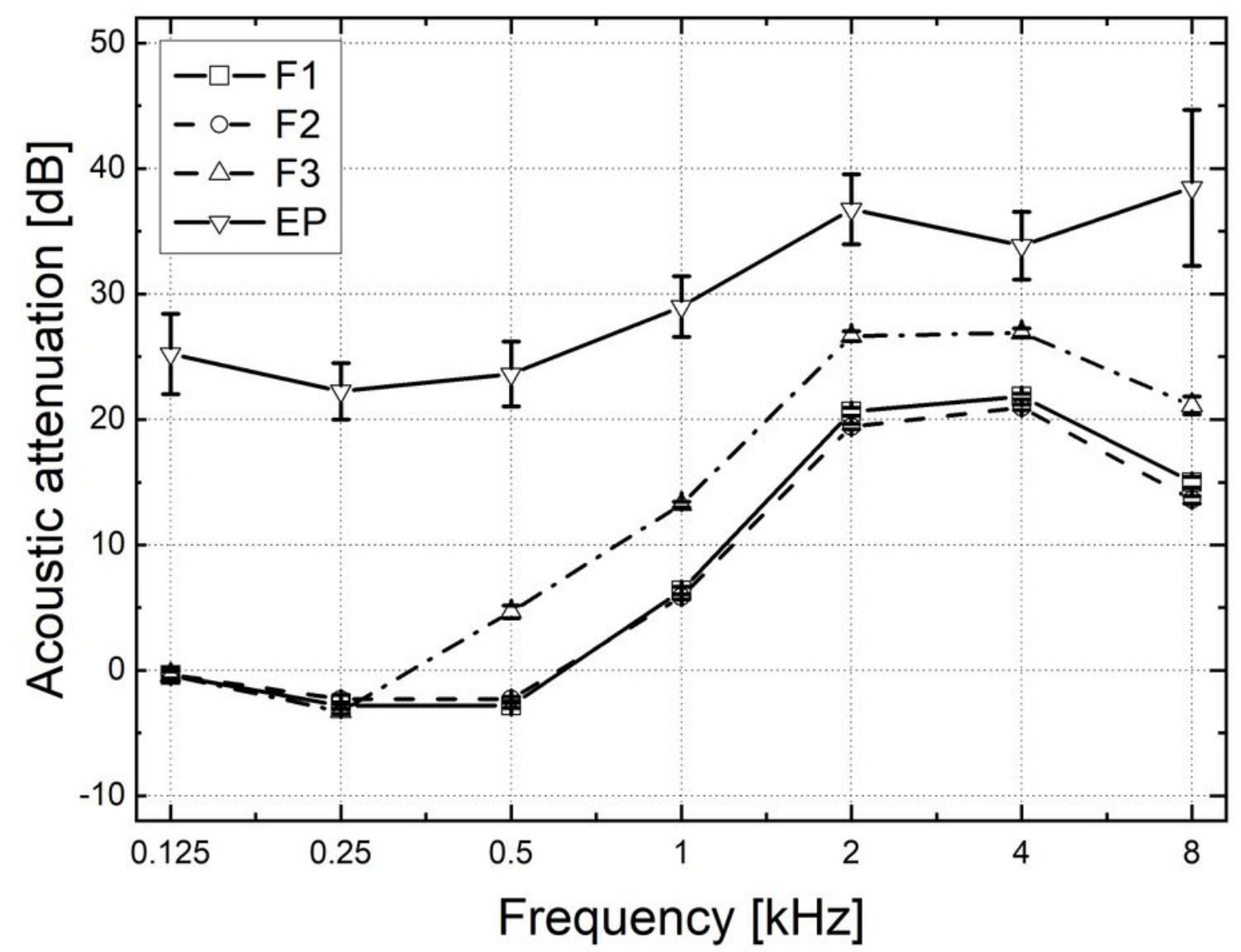

Figure 16

Mean values of attenuation as a function of frequency for earplugs with F1, F2 and F3 filter and for full earplug. Results obtained using the subjective method. Error bars show 95\% confidence interval 


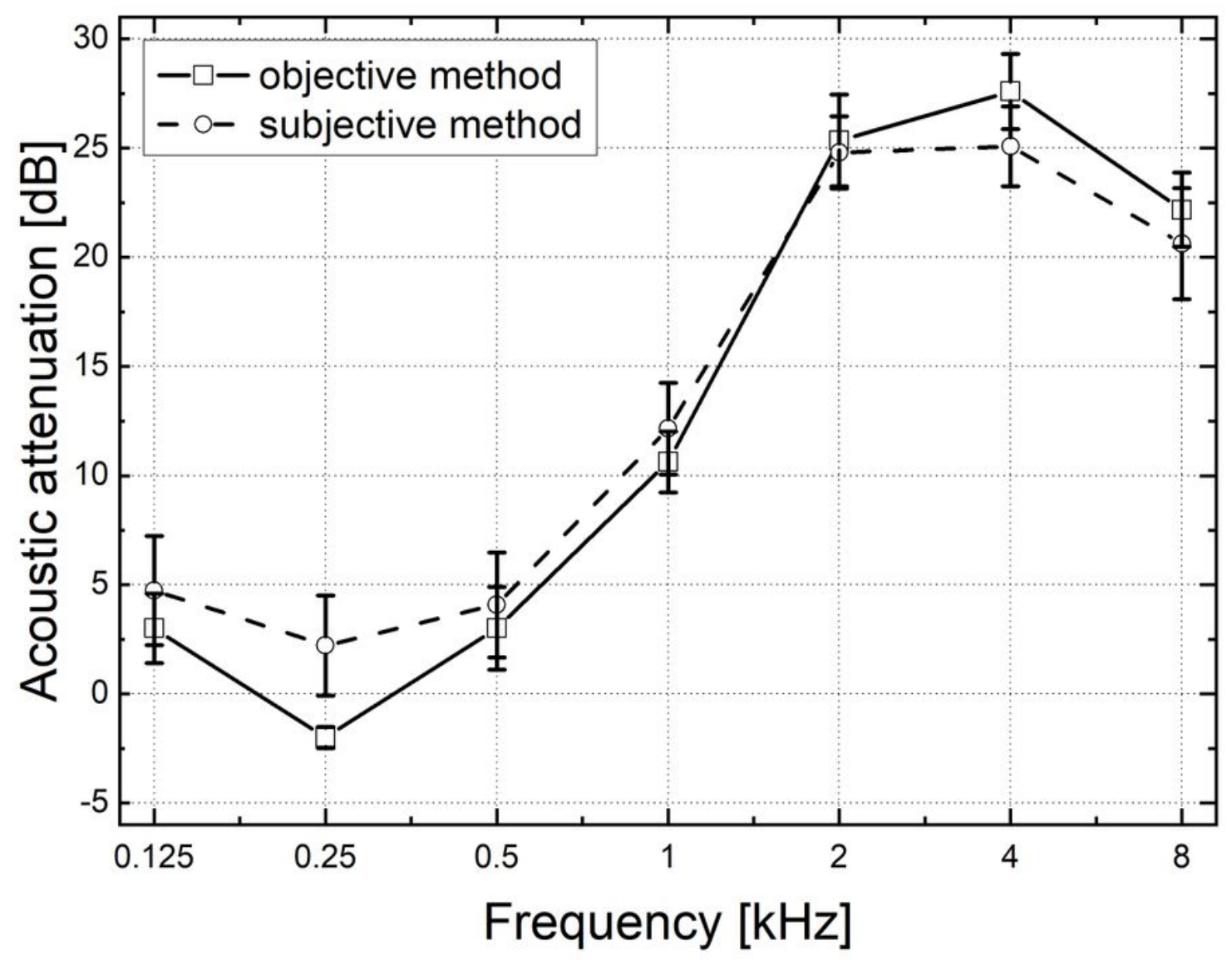

Figure 17

Mean values of attenuation depending on the frequency obtained with the objective method (empty squares) and the subjective method (empty circles). Error bars show a $95 \%$ confidence interval 


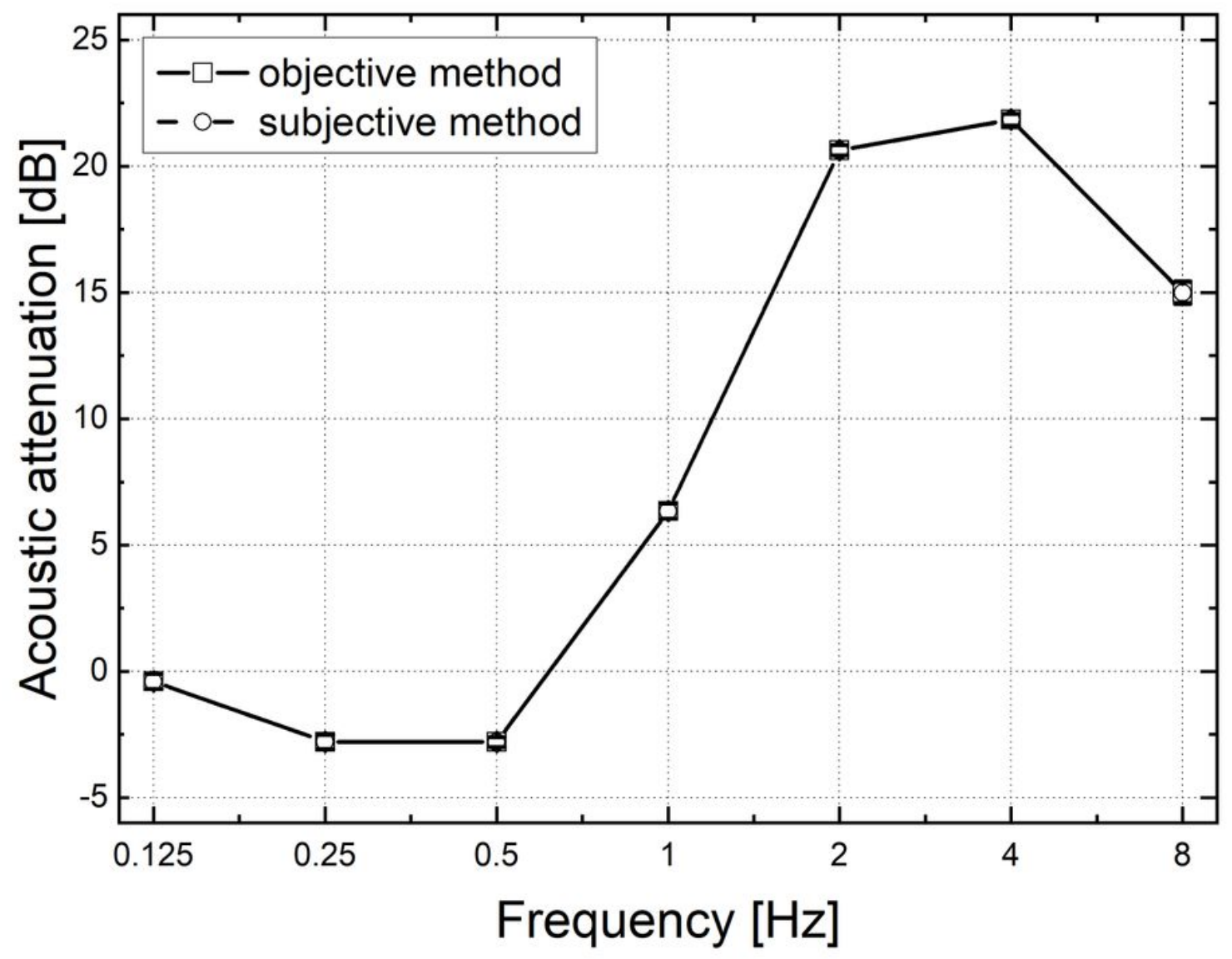

Figure 18

Mean values of attenuation for the earplug with filter F1 depending on the frequency obtained in the objective method (empty squares) and the subjective method (empty circles). Error bars show a 95\% confidence interval 


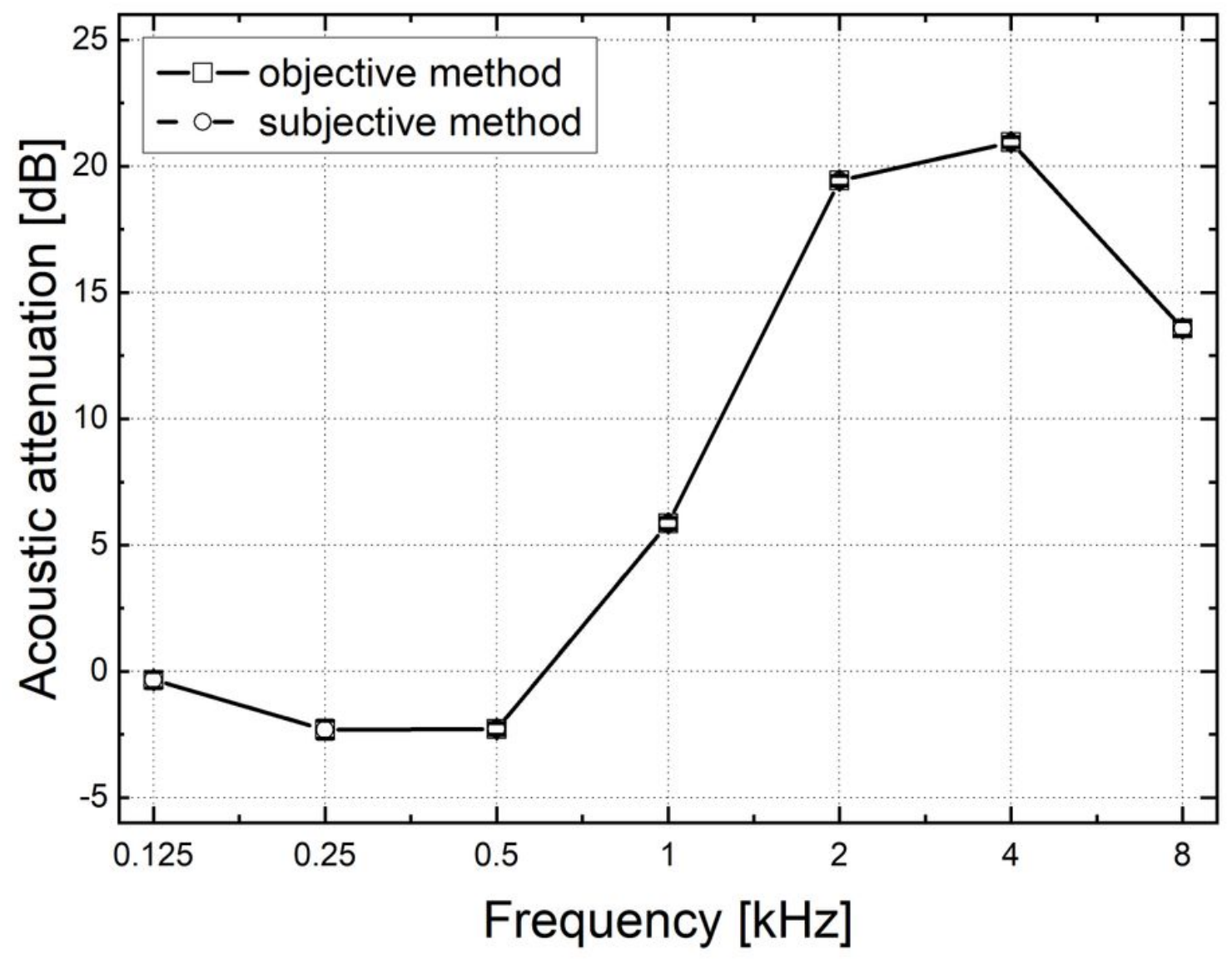

Figure 19

Mean values of attenuation for the earplug with filter F2 depending on the frequency obtained in the objective method (empty squares) and the subjective method (empty circles). Error bars show a 95\% confidence interval 


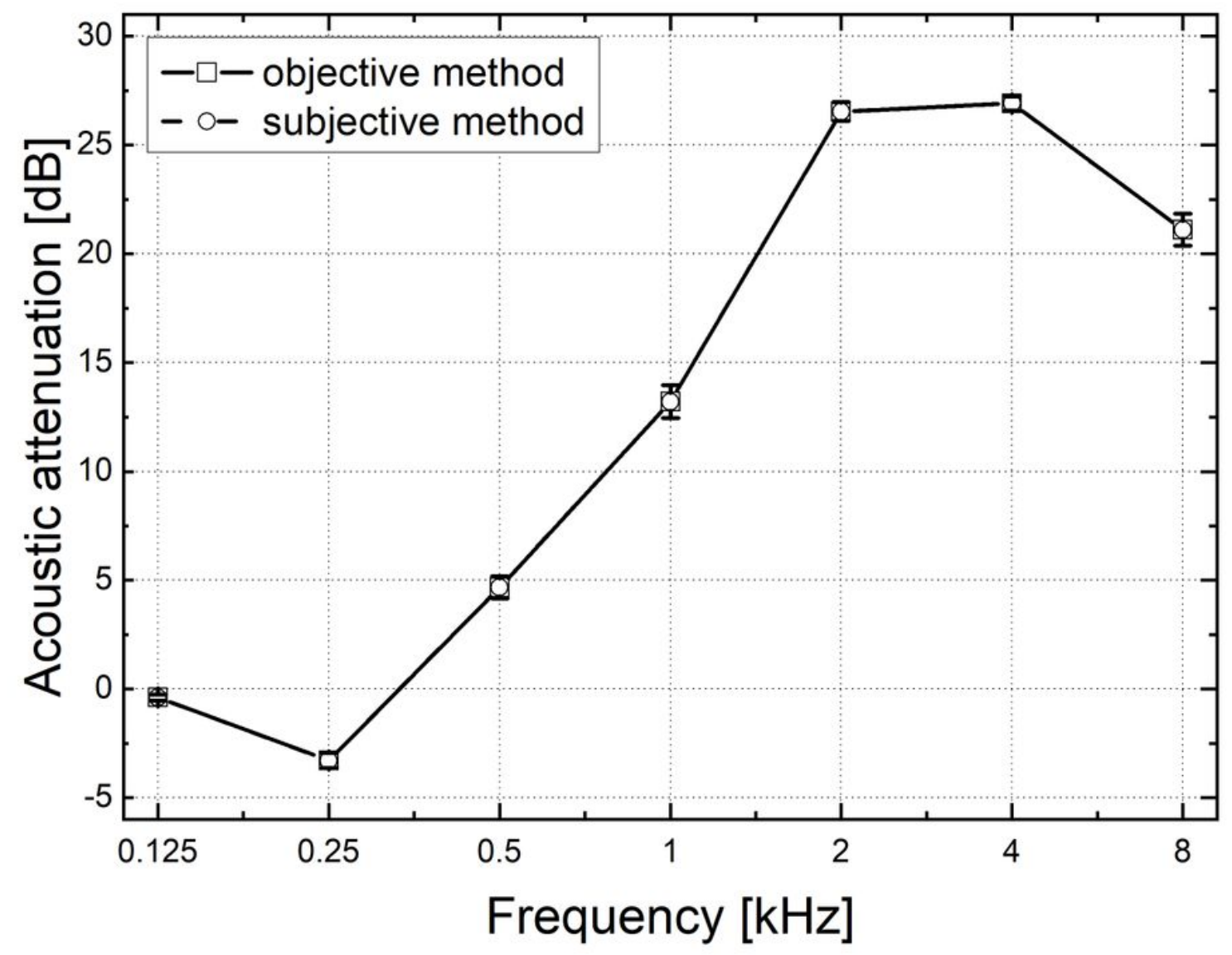

Figure 20

Mean values of attenuation for the earplug with filter F3 depending on the frequency obtained in the objective method (empty squares) and the subjective method (empty circles). Error bars show a 95\% confidence interval 


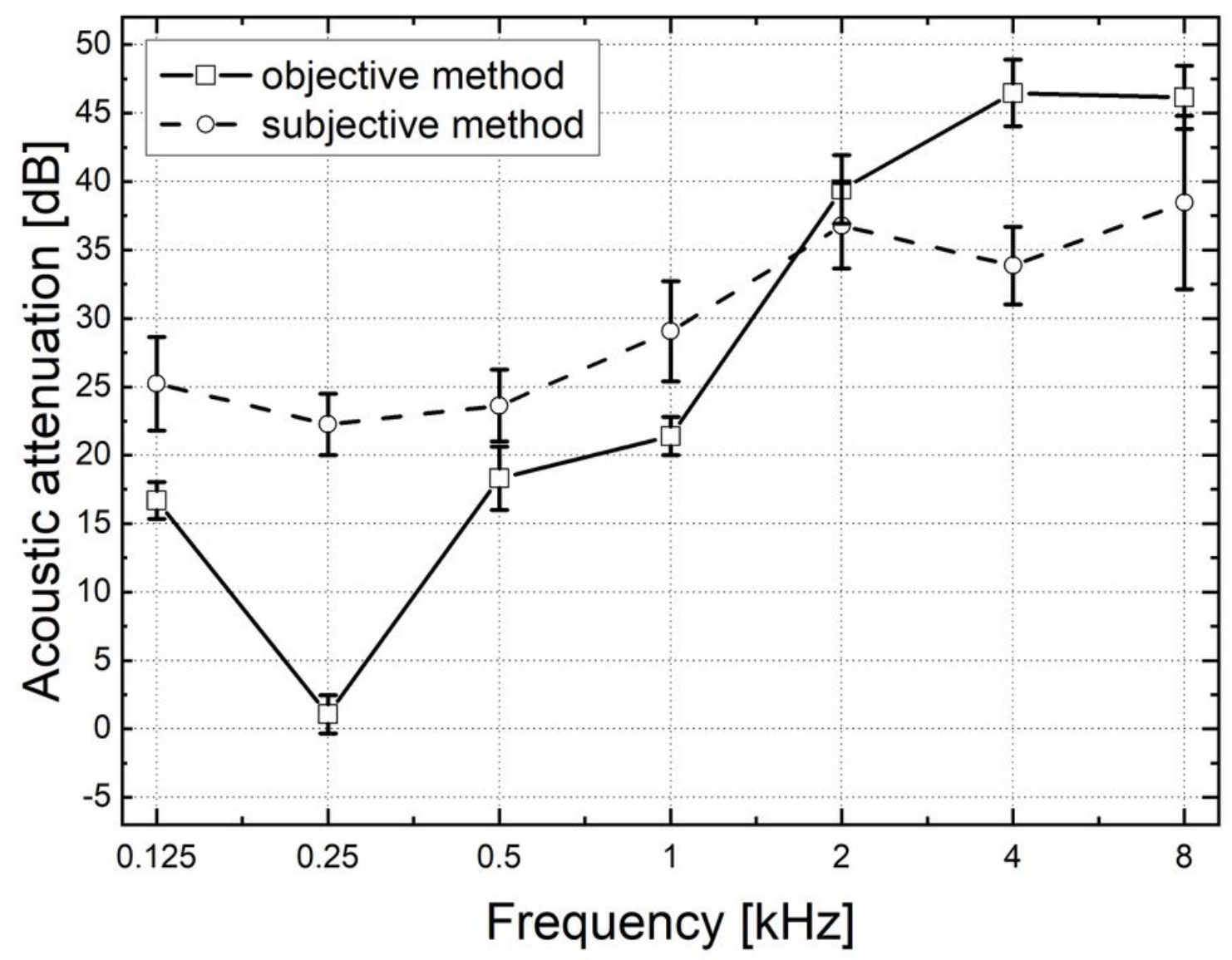

Figure 21

Mean values of attenuation for the full earplug depending on the frequency obtained in the objective method (empty squares) and the subjective method (empty circles). Error bars show a $95 \%$ confidence interval 